\title{
About Partial Reachability Issues in an SEIR Epidemic Model and Related Infectious Disease Tracking in Finite Time under Vaccination and Treatment Controls
}

\author{
Manuel De la Sen $\left(\mathbb{D},{ }^{1}\right.$ Asier Ibeas, ${ }^{2}$ and Raul Nistal ${ }^{1}$ \\ ${ }^{1}$ Institute of Research and Development of Processes (IIDP), University of the Basque Country, Campus of Leioa, P.O. Box 48940, \\ Leioa, Bizkaia, Spain \\ ${ }^{2}$ Department of Telecommunications and Systems Engineering, Universitat Autònoma de Barcelona UAB, \\ 08193 Barcelona, Spain
}

Correspondence should be addressed to Manuel De la Sen; manuel.delasen@ehu.eus

Received 5 January 2021; Revised 28 January 2021; Accepted 4 February 2021; Published 1 March 2021

Academic Editor: Ya Jia

Copyright (c) 2021 Manuel De la Sen et al. This is an open access article distributed under the Creative Commons Attribution License, which permits unrestricted use, distribution, and reproduction in any medium, provided the original work is properly cited.

\begin{abstract}
This paper studies some basic properties of an SEIR (Susceptible-Exposed-Infectious-Recovered) epidemic model subject to vaccination and treatment controls. Firstly, the basic stability, boundedness, and nonnegativity of the state trajectory solution are investigated. Then, the problem of partial state reachability from a certain state value to a targeted one in finite time is focused on since it turns out that epidemic models are, because of their nature, neither (state) controllable from a given state to the origin nor reachable from a given initial condition. The particular formal statement of the partial reachability is focused on as a problem of output-reachability by defining a measurable output or lower dimension than that of the state. A special case of interest is that when the output is defined as the infectious subpopulation to be step-to-step tracked under suitable amounts being compatible with the required constraints. As a result, and provided that the output-controllability Gramian is nonsingular on a certain time interval of interest, a feedback control effort might be designed so that a prescribed value of the output can be approximately tracked. A linearization approximation is performed to simplify and facilitate the above task which is based on a point-to-point linearization of the solution trajectory. To this end, an "ad hoc" sampled approximate output trajectory is defined as control objective to be targeted through a point-wise calculated Jacobian matrix. A supervised appropriate restatement of the targeted suited sampled output values is redefined, if necessary, to make the initial proposed sampled trajectory compatible with the various needed constraints on nonnegativity and control boundedness. The design can be optionally performed under constant or adaptive sampling rates. Finally, some numerical examples are given to test the theoretical aspects and the design efficiency of the model.
\end{abstract}

\section{Introduction}

Epidemic mathematical models are receiving very significant attention in the last decades due to their inherent interest to predict the progression of the force intensity of the infections through time so as to take appropriate decisions about how to mitigate them and how to conduct in an adequate way the hospital means requirements to fight against them. In this context, it is very important to study the influence in the disease evolution of potential control actions like typically the vaccination and treatment controls and to accommodate their convenient application to items like their availability and economic costs through time and their demand in view of the disease progression. In this context, a relevant part of the available background literature has paid attention to their optimal management and application to the population. See, for instance, [1-9] and some of the references therein. On the other hand, it can be also pointed out that 
quarantine actions for the removal of some healthy susceptible individuals from the infective environment can be interpreted as an impulsive vaccination in continuous-time models, while a similar strategy on the infectious ones is in fact performed through an impulsive antiviral or antibiotic control. See, for instance, [8-10] and some of the references therein. In particular, a modified SEIR model which adds a new infectious population (that is, the lying infective corpses) is considered, so-called the SEIADR model, and, apart from the vaccination and treatment controls, an impulsive control is applied to this population which is interpreted as the recovery from the streets of the lying infective corpses by ad hoc organizes brigades or volunteers when necessary. In this way, the SEIADR model is subject to three different controls. The way of updating the feedback controller gains is supervised by an ad hoc technique proposed in [9] for the discrete version of the SEIADR which can manage a trade-off between the infection evolution and the vaccinations costs. Besides, the epidemic models can be extended for use by being split into different coupled nodes to describe interacting populations among different towns or regions. See, for instance, [11-15] and related background bibliography. The design of observers to estimate the model state when it is not fully measurable or perfectly known has also received significant attention in several papers, for instance, in [16]. Other topics, like some cases of the relevance of complex dynamics or the influence of pathogens in certain epidemic models, such as ticks and mites, have been also focused on and studied. See, for instance, [17-19] and references therein. The approximate controllability and reachability problems in time-varying systems have been considered in [20], and some ad hoc examples related to epidemic models have been given, while in [21] the classical hyperstability theory of control systems has been adapted to be used in derivation of very wide structure types for nonlinear feedback rules of vaccination and treatment controls in some epidemic models. The design of control laws for certain ecology models, like Beverton-Holt equation or epidemic models, has received significant attention in the literature, in particular, the monitoring of the carrying capacity in aquaculture or the updated design of the feedback gains of vaccination and treatment control. See, for instance, $[8,11,13,14]$ and $[21,22]$. On the other hand, epidemic models based on differential equations, as other biological and in general controlled systems, can be discretized in order to facilitate their computational descriptions. See, for instance, [23-30] and some of the references therein. It has to be pointed out that the stochastic formalism has been also seen to be useful in studying some concerns in the epidemic models behavior, typically, the influences of perturbations in the equilibrium points and uncertainties in the data. See, for instance, [31, 32] and some references therein. Moreover, the relevance of modeling small communities can be useful for interpreting the disease evolution or taking intervention decisions since the total population is an aggregation of smaller communities [33].

This paper proposes a formal statement of partial reachability stated as one of output-reachability by defining a measurable output or lower dimension than that of the state on a SEIR-type epidemic model. The main reason is that epidemic models are neither controllable to the origin nor reachable, because of their nature, since the controls always transfer vaccinated susceptible individuals and treated infectious ones to the recovered subpopulation so that the whole subpopulations cannot be jointly prefixed as separate objectives to be tracked at any finite time through control actions. This would happen even under the unrealistic and unfeasible absence of constraints of control boundedness type and of nonnegativity of the state trajectory solution. A special case of interest focused on is when the output is defined as the infectious subpopulation to be step-to-step tracked under suitable amounts being compatible with the required constraints. A point-to-point linearization approximation of the sampled output solution trajectory is performed as a preparatory step for the approximate reachability design of a suited output trajectory though the vaccination and treatment controls. A reformulation of the output tracking objectives is performed in a higher-level design step, if necessary, in order to satisfy the solution nonnegativity and control boundedness constraints. Several alternative designs are discussed based either on the use of constant sampling rates or on the choice of time-varying sampling periods which are adapted to the variation slope of the output through time.

The paper is organized as follows. Section 2 describes the proposed SEIR epidemic model which is eventually subject to vaccination and treatment controls. Its properties of solution boundedness for finite initial conditions, the solution nonnegativity for all time under any nonnegative initial conditions, and its equilibrium points are investigated in Section 3. Section 4 is devoted to the study of the output-reachability aspects of a special model of the previous general one which consider the recruitment as dependent on the birth rate and inward/outward flex rates. The output is defined with a lower dimensionality than that of the state model $(n=4)$, which is a necessary condition for the approximate output-reachability of the model, since the system is not reachable, and it can typically be chosen, in particular, as the infectious subpopulation through time. The output solution is linearized via an updated sampled Jacobian matrix through a set of samples. Such samples can be selected with a constant sampling period or with a time-varying one which is obtained from a simple adaptive sampling rule which takes into account the timederivative values of the output. Section 5 presents and discusses numerical examples to illustrate the previous theoretical aspects of the paper. Finally, some conclusions are given in Section 6.

\section{The SEIR Model with Vaccination and Treatment Controls}

Consider the following SEIR (Susceptible-S, Exposed-E, Infectious-I, Recovered-R) epidemic model subject to vaccination and treatment controls:

$$
\begin{aligned}
& \dot{S}(t)=b N(t)-d S(t)-c S(t) I(t)-V(t), \\
& \dot{E}(t)=c S(t) I(t)-(f+d) E(t),
\end{aligned}
$$




$$
\begin{aligned}
& \dot{I}(t)=f E(t)-(g+a+d) I(t)-T(t), \\
& \dot{R}(t)=g I(t)-d R(t)+V(t)+T(t),
\end{aligned}
$$

$\forall t \in \mathbf{R}_{0+}=\mathbf{R}_{+} \cup\{0\}$, subject to initial conditions $S(0)=S_{0}$, $E(0)=E_{0}, \quad I(0)=I_{0}$, and $R(0)=R_{0}$ with $\min \left(S_{0}, E_{0}\right.$, $\left.I_{0}, R_{0}\right) \geq 0$ and $S_{0}+E_{0}+I_{0}+R_{0}>0$, where $S(t), E(t), I(t)$, and $R(t)$ are, respectively, the susceptible, exposed, infectious, and recovered subpopulations and $V(t)$ and $T(t)$ are, respectively, the vaccination and treatment control interventions on the susceptible and infectious, respectively, used to decrease the infection progression.

The parameterization of the above model is as follows:

$c$ is the transmission rate of the disease

$b$ is the natural birth date

$a$ is the death rate caused by the disease

$d$ is the natural death rate

$f$ is the rate of transfer of exposed individuals to infectious ones

$g$ is the natural recovery rate.

Summing up the four subpopulations (1)-(4), one gets the total population $N(t)=S(t)+E(t)+I(t)+R(t)$, $\forall t \in \mathbf{R}_{0+}$ which satisfies the subsequent differential equation:

$$
\dot{N}(t)=(b-d) N(t)-a I(t), \quad \forall t \in \mathbf{R}_{0+},
$$

with $N(0)=N_{0}=S_{0}+E_{0}+I_{0}+R_{0}$. A closer model to the above one without treatment control has been discussed in [8] in the context of imposing upper-bound constraints on the number of available vaccines at any time instant when mixed state control constraints are set. The possibility of establishing upper-bounds on the number of the susceptible with and without bounds on the availability of vaccines has been also explored.

Some of the main theoretical and numerical results of this paper rely on the use of linear feedback vaccination and treatment controls which translate into feedback actions for the respective efforts which are proportional to the susceptible and infectious subpopulations, respectively. The feedback control techniques are very common in the background literature including that referring to disease spread. For instance, the vaccination rate is a control feedback gain invoked in $[1-3,5,9-11,13,14,21]$, some references therein, and others from related literature which use the feedback information for the implementation of the vaccination campaigns. In this way, the vaccination effort is proportional to the number of susceptible individuals, and this allows for intensifying the effort when the disease spread is high and to decrease it otherwise. In the same way, treatment efforts can be generated via feedback on the basis that slightly infectious and asymptomatic individuals, who are relevant fractions of the infectious total population in some diseases, do not usually require hospital treatment.

\section{Discussion of the Main Properties of the Epidemic Model}

This section states and proves the main properties of nonnegativity and stability of the above epidemic model. The concepts of epidemiology are merged with those of the mathematical modeling and dynamical systems. Therefore, the terminology of these mentioned fields is equivalently used throughout this section in order to present the techniques employed within the infectious diseases spreading description frame.

The subsequent first result relies on the nonnegativity of all the subpopulations for all time under any given nonnegative initial conditions and under nonnegativity and upper-bounding conditions of the control efforts. The nonnegativity of the state trajectory solution of (1)-(4), i.e., the nonnegativity of all the subpopulations for all time in terms of the epidemic diseases terminology, holds irrespective of the subpopulations being bounded or not. The proof is made by inspecting directly the solutions of the subpopulations from their respective differential equations instead of inspecting the zero crossings of the corresponding time-derivatives. If, in addition, $b \leq d$, then all the subpopulations are bounded for all time. This follows from the uniform boundedness of the total population together with the nonnegativity through time of all the subpopulations.

Theorem 1. The trajectory solution of (1) is nonnegative for all time for any given nonnegative initial conditions provided that the control efforts are sufficiently small for all time according to the constraints $0 \leq V(t) \leq b N(t)$ and $0 \leq T(t) \leq f E(t)$; $\forall t \in \mathbf{R}_{0+}$. If, in addition, $b \leq d$, then all the subpopulations and then the total population are bounded for all time. 
Proof. Note from (1) that

$$
S(t)=e^{-\left(\mathrm{d} t+c \int_{0}^{t} I(\tau) \mathrm{d} \tau\right)}\left[S_{0}+\int_{0}^{t} e^{\left(\mathrm{d} \tau+c \int_{0}^{\tau} I(\sigma) \mathrm{d} \sigma\right)}(b N(\tau)-V(\tau)) \mathrm{d} \tau\right] \geq 0, \quad \forall t \in \mathbf{R}_{0+}
$$

since $S_{0} \geq 0$ and $V(t) \leq b N(t), \forall t \in \mathbf{R}_{0+}$. Now, note from (3) that $\dot{I}(t) \geq-(g+a+d) I(t), \forall t \in \mathbf{R}_{0+}$, since $T(t) \leq f E(t)$. Then, there exists a function $\varepsilon_{I}: \mathbf{R}_{0+} \longrightarrow \mathbf{R}_{0+}$ defined by $\varepsilon_{I}(t)=f E(t)-T(t)$, such that $\dot{I}(t)=-(g+a+d)$ $I(t)+\varepsilon_{I}(t), \forall t \in \mathbf{R}_{0+}$, so that

$$
\begin{aligned}
I(t) & =e^{-(g+a+d) t}\left(I_{0}+\int_{0}^{t} e^{(g+a+d) \tau}(f E(\tau)-T(\tau)) \mathrm{d} \tau\right) \\
& \leq e^{-(g+a+d) t} I_{0}+\int_{0}^{t} e^{-(g+a+d)(t-\tau)} \varepsilon_{I}(\tau) \mathrm{d} \tau \geq 0, \quad \forall t \in \mathbf{R}_{0+},
\end{aligned}
$$

since $I_{0} \geq 0$. Furthermore, one has from (2) that

$$
E(t)=e^{-(f+d) t} E_{0}+c \int_{0}^{t} e^{-(f+d)(t-\tau)} S(\tau) I(\tau) \mathrm{d} \tau \geq 0, \quad \forall t \in \mathbf{R}_{0+},
$$

since $E_{0} \geq 0$ and $S(t) I(t) \geq 0, \forall t \in \mathbf{R}_{0+}$, from (6) and (7). Finally, one gets from (4) that

$$
R(t)=e^{-\mathrm{d} t} R_{0}+\int_{0}^{t} e^{-\mathrm{d}(t-\tau)}(g I(\tau)+V(\tau)+T(\tau)) \mathrm{d} \tau \geq 0, \quad \forall t \in \mathbf{R}_{0+},
$$

since $R_{0} \geq 0$ and $g I(t)+V(t)+T(t) \geq 0, \forall t \in \mathbf{R}_{0+}$, from the assumptions on the controls and (7). It has been proved that all the subpopulations (and thus the total one) are nonnegative for all time so that the total population is also bounded for all time. Thus, one has from (5) that if $b \leq d$, then one obtains from (5) that $0 \leq N(t)=e^{(b-d) t} N_{0}$ $-a \int_{0}^{t} e^{(b-d)(t-\tau)} I(\tau) \mathrm{d} \tau \leq N_{0}, \quad \forall t \in \mathbf{R}_{0+}$. Accordingly, the total population is bounded for all time and any finite initial conditions. Since all the subpopulations are nonnegative for all time, all the subpopulations are also bounded for all time.

It can be also allowed to violate the upper-bounds of the vaccination and treatment controls beyond the time intervals where they hold while keeping the nonnegativity of the solutions. This holds under certain trade-offs among previously reached positive values of the susceptible and infectious, respectively; the deviation of the corresponding upperbounds and the time intervals along those violations stand. One has the following subsequent related simple result.

Corollary 1. Assume that $0 \leq V(t) \leq b N(t)$ for $t \in[0, T]$ and that $V(t)>b N(t), \forall t \in\left(T, T_{1}\right]$, with $T \leq T_{1}=T_{1}(T)=$ $\max \arg \left(r \in \mathbf{R}_{0+}:(r \geq T) \wedge\left(\int_{T}^{r} e^{\left(d \tau+c \int_{0}^{\tau} I(\sigma) d \sigma\right)} \mid b N(\tau)-V(\tau)\right.\right.$ $\mid d \tau) \leq S(T))$. Then, $S(t) \geq 0, \forall t \in\left[0, T_{1}\right]$.

Assume that $0 \leq T(t) \leq e E(t)$ for $t \in[0, T]$ and that $T(t)>f E(t), \forall t \in\left(T, T_{2}\right]$, with $T \leq T_{2}=T_{2}(T)=\max$ arg $\left(r \in \mathbf{R}_{0+}:(r \geq T) \wedge\left(\int_{T}^{r} e^{(g+a+d) \tau}|f E(\tau)-T(\tau)| \mathrm{d} \tau\right) \leq I(T)\right)$. Then, $I(t) \geq 0 ; \forall t \in\left[0, T_{2}\right]$.

Proof. One has from Theorem 1 that $S(t) \geq 0, \forall t \in[0, T]$, since $0 \leq V(t) \leq b N(t)$ for $t \in[0, T]$. In addition, one has from (6) by retaking initial conditions at $t=T$ that if $V(t)>b N(t), \forall t \in\left(T, T_{1}\right]$, then

$$
S(t) \geq e^{-\left(\mathrm{d}\left(T_{1}-T\right)+c \int_{T}^{T_{1}} I(\tau) \mathrm{d} \tau\right)}\left(S(T)-\int_{T}^{T_{1}} e^{\left(\mathrm{d} \tau+c \int_{0}^{\tau} I(\sigma) \mathrm{d} \sigma\right)}|b N(\tau)-V(\tau)| \mathrm{d} \tau\right) \geq 0, \quad \forall t \in\left(T, T_{1}\right]
$$

As a result, $S(t) \geq 0, \forall t \in\left[0, T_{1}\right]$, from (10). On the other hand, one has from Theorem 1 that $I(t) \geq 0, \forall t \in[0, T]$, since $0 \leq T(t) \leq f E(t)$ for $t \in[0, T]$. Besides, one has from
(2) by retaking initial conditions at $t=T$ that if $T(t)>f E(t), \forall t \in\left(T, T_{2}\right]$, then

$$
I(t) \geq e^{-(g+a+d)\left(T_{2}-T\right)}\left(I(T)-\int_{T}^{T_{2}} e^{(g+a+d) \tau}|f E(\tau)-T(\tau)| \mathrm{d} \tau\right) \geq 0, \quad \forall t \in\left(T, T_{1}\right]
$$

and $I(t) \geq 0, \forall t \in\left[0, T_{2}\right]$, from (11).

The subsequent result is concerned with proving that for $b<d$ and for $b=d$ with $a>0$, a globally asymptotically stable disease-free equilibrium point exists under the control constraints of Theorem 1 . It is also proved that all the subpopulations are asymptotically unbounded if $b>d$. 
Theorem 2. The following properties hold:

(i) If the control constraints of Theorem 1 hold and $b<d$, then a globally asymptotically stable disease-free equilibrium point implying also asymptotic extinction (that is, the total subpopulation asymptotically extinguishes for any finite initial conditions) exists.

If $b=d, a \neq 0$, and the vaccination control converges asymptotically to $V_{d f}^{*}$, then a disease-free equilibrium point, which does not imply asymptotic extinction, exists, and the treatment control converges asymptotically to zero. The disease-free equilibrium point is defined by $S_{d f}^{*}=\left(b N_{d f}^{*}-V_{d f}^{*}\right) / d, \quad E_{d f}^{*}=I_{d f}^{*}=0, \quad T_{d f}^{*}=0$, $R_{d f}^{*}=\left(V_{d f}^{*} / d\right)$ giving a total equilibrium population $N_{d f}^{*}=S_{d f}^{*}+R_{d f}^{*}=N_{0}-a \int_{0}^{\infty} I(\tau) d \tau$, and $I(t)$ is integrable on $[0, \infty]$. If the vaccination and treatment controls are generated by linear feedback of the susceptible and infectious, respectively, that is, $V(t)=k_{V} S(t)$ and $T(t)=k_{I} I(t)$, then $S_{d f}^{*}=b N_{d f}^{*} /\left(b+k_{V}\right)$ and $R_{d f}^{*}=$ $\left(k_{V} S_{d f}^{*} / d\right)=\left(k_{V} b N_{d f}^{*} / \quad d\left(d+k_{V}\right)\right)=k_{V} N_{d f}^{*} /(b$ $\left.+k_{V}\right)$, where $V_{d f}^{*}=k_{V} S_{d f}^{*}$ and $T_{d f}^{*}=k_{T} I_{d f}^{*}$.

(ii) If $b>d$, then $N(t) \longrightarrow \infty$ as $t \longrightarrow \infty$ for any nontrivial solution. If, furthermore, $\sup _{t \in \mathbf{R}_{0+}} V(t) \leq$ $V_{0}<\infty$ and $\sup _{t \in \mathbf{R}_{0+}} T(t) \leq T_{0}<\infty$, then the infection becomes asymptotically unbounded and all the subpopulations become asymptotically unbounded for any nonzero initial conditions.

Proof. One obtains from (5) that

$N(t)=e^{(b-d) t} N_{0}-a \int_{0}^{t} e^{(b-d)(t-\tau)} I(\tau) \mathrm{d} \tau, \quad \forall t \in \mathbf{R}_{0+}$.

Then, one has the following:

(a) If $0 \leq V(t) \leq b N(t), 0 \leq T(t) \leq f E(t), \forall t \in \mathbf{R}_{0+}$, and $b<d$, then $N(t) \longrightarrow 0$ as $t \longrightarrow \infty$ so that $S(t) \longrightarrow 0, E(t) \longrightarrow 0, I(t) \longrightarrow 0$, and $R(t) \longrightarrow 0$ as $t \longrightarrow \infty$ irrespective of the initial conditions, since from Theorem 1 all the subpopulations and the total population are nonnegative for all time. As a result, the unique equilibrium point is a globally stable disease-free one implying total extinction; that is, $\quad x_{d f}^{*}=\left(S_{d f}^{*}, E_{d f}^{*}, I_{d f}^{*}, R_{d f}^{*}\right)^{T}=(0,0,0,0)^{T}$. Besides, from the constraints of Theorem 1, $V(t) \longrightarrow V_{d f}^{*}=0, T(t) \longrightarrow T_{d f}^{*}=0$ as $t \longrightarrow \infty$.

(b) If $b=d$ and $a \neq 0$, then one has from (12) and Theorem 1 that

$$
\infty>N(t)=N_{0}-a \int_{0}^{t} I(\tau) \mathrm{d} \tau \geq 0, \quad \forall t \in \mathbf{R}_{0+} .
$$

Then, $\quad \int_{0}^{t} I(\tau) \mathrm{d} \tau \leq\left(N_{0} / a\right), \quad \forall t \in \mathbf{R}_{0+}, \quad \int_{0}^{\infty} I(\tau) \mathrm{d} \tau \leq$ $\left(N_{0} / a\right)$, and $\lim _{t \rightarrow \infty} I(t)=0$ irrespective of the initial conditions. Since $I(t)=I_{0}+\int_{0}^{t} \dot{I}(\tau) \mathrm{d} \tau \longrightarrow 0$ as $t \longrightarrow \infty$, then $\lim _{t \rightarrow \infty} \int_{0}^{t} \dot{I}(\tau) \mathrm{d} \tau=-I_{0}<+\infty$ so that; since
$\dot{I}: \mathbf{R}_{0+} \longrightarrow \mathbf{R}$ is uniformly continuous from the differential equation (2) and $T: \mathbf{R}_{0+} \longrightarrow \mathbf{R}_{0+}$ is uniformly continuous, then $\lim _{t \rightarrow \infty} \dot{I}(t)=0$ from Barbalat's lemma. Now, $I(t) \longrightarrow I_{d f}^{*}=0$ and $\dot{I}(t) \longrightarrow 0$ so that $E(t) \longrightarrow E_{d f}^{*}=0$; then, $T(t) \longrightarrow T_{d f}^{*}=0$ (from the control constraint of Theorem 1) as $t \longrightarrow \infty$ irrespective of the initial conditions.

Thus, one gets from (4) that $R(t) \longrightarrow R_{d f}^{*}=\left(V_{d f}^{*} / d\right)$ as $t \longrightarrow \infty$ since $V(t) \longrightarrow V_{d f}^{*}$ as $t \longrightarrow \infty$. Therefore, $(N(t)-S(t)-R(t)) \longrightarrow\left(N(t)-S(t)-R_{d f}^{*}\right) \longrightarrow 0 \quad$ as $t \longrightarrow \infty$. From (13), and since $I(t)(\geq 0) \longrightarrow 0$ as $t \longrightarrow \infty$, the total population has a limit as time tends to infinity, that is, $N(t) \longrightarrow \infty$ as $t \longrightarrow \infty$. Then, note that $S_{d f}^{*}$ is not determined from (1) since $b=d, E_{d f}^{*}=I_{d f}^{*}=0$, and $R_{d f}^{*}=\left(V_{d f}^{*} / d\right)$. However, such a limit $S_{d f}^{*}$ has to exist being nonnegative and finite from $S(t) \longrightarrow N_{0}-a \int_{0}^{\infty} I(\tau) \mathrm{d} \tau-$ $\left(V_{d f}^{*} / d\right)$ as $t \longrightarrow \infty$, since $\int_{0}^{\infty} I(\tau) \mathrm{d} \tau \leq\left(N_{0} / a\right)$ and $I(t)(\geq 0) \longrightarrow 0$ as $t \longrightarrow \infty$ implies that $\int_{0}^{\infty} I(\tau) \mathrm{d} \tau=L_{I}$ exists and it is a nonnegative finite number for any given finite initial conditions. Then,

$$
S(t) \longrightarrow S_{d f}^{*}=N_{0}-a L_{I}-\frac{V_{d f}^{*}}{d},
$$

with $0 \leq L_{I} \leq\left(\left(N_{0}-V_{d f}^{*} / d\right) / a\right)$ so that a globally asymptotically stable disease-free equilibrium point still exists if $b=d$. In this case, one gets directly that $S_{d f}^{*}=\left(b N_{d f}^{*}-\right.$ $\left.V_{d f}^{*}\right) / b$ from (1) if $I_{d f}^{*}=0, E_{d f}^{*}=T_{d f}^{*}=0$ from (2) and (3) and $R_{d f}^{*}=\left(V_{d f}^{*} / b\right)$ from (4). One also gets from (5) that $N_{d f}^{*}=S_{d f}^{*}+R_{d f}^{*}=N_{0}-a \int_{0}^{\infty} I(\tau) \mathrm{d} \tau$ and $I(t)$ is integrable on $[0, \infty]$. If the vaccination and treatment controls are generated by linear feedback of the susceptible and infectious, respectively, that is, $V(t)=k_{V} S(t)$ and $T(t)=k_{I} I(t)$, then $\quad S_{d f}^{*}=b N_{d f}^{*} /\left(b+k_{V}\right) \quad$ and $\quad R_{d f}^{*}=\left(k_{V} S_{d f}^{*} / b\right)=$ $k_{V} N_{d f}^{*} /\left(b+k_{V}\right)$ since $b=d$, where $V_{d f}^{*}=k_{V} S_{d f}^{*}$ and $T_{d f}^{*}=k_{T} I_{d f}^{*}$. Besides, $\lim _{t \rightarrow \infty} N(t)=N_{d f}^{*}=a \int_{0}^{\infty} I(\tau) \mathrm{d} \tau$. Property (i) has been proved.

To prove Property (ii), assume that $b>d$. Then, one has from (5) and the nonnegativity of the solution from Theorem 1 , since $N(t) \geq I(t), \forall t \in \mathbf{R}_{0+}$, that

$$
\begin{aligned}
N(t)= & N_{0}+\int_{0}^{t}[(b-d) N(\tau)-a I(\tau)] \mathrm{d} \tau \geq N_{0} \\
& +\int_{0}^{t}(b-d-a) I(\tau) \mathrm{d} \tau .
\end{aligned}
$$

Assume that lim $\sup _{t \rightarrow \infty} \sup _{0 \leq \tau \leq t} N(t)<\infty$ and proceed by contradiction arguments. One has from (15) that two cases can arise, namely:

Case (i): assume that $b>d+a$; then, since limsup $\sup _{t \rightarrow \infty}$ $\sup _{0 \leq \tau \leq t} N(t)<\infty$, this implies from (15) that $\lim _{t \rightarrow \infty} I(t)=0$. One also gets from (5) that $\dot{N}(t)=(b-d) N(t)-a I(t) \geq a(N(t)-I(t)), \forall t \in \mathbf{R}_{0+}$,

$$
\infty>\frac{1}{a}\left(N(t)-N_{0}\right)=\int_{0}^{t}(N(\tau)-I(\tau)) \mathrm{d} \tau>0, \quad \forall t \in \mathbf{R}_{+},
$$


and $(N(t)-I(t)) \longrightarrow 0$ as $t \longrightarrow \infty$ if $N_{0}>I_{0} \geq 0$. Since $I(t) \longrightarrow 0$ as $t \longrightarrow \infty$, then $N(t) \longrightarrow 0$ as $t \longrightarrow \infty$. However, then, one gets the contradiction to the assumption $\quad \lim \sup _{t \rightarrow \infty} \sup _{0 \leq \tau \leq t} N(t)<$ $\infty, 0 \leq \lim _{t \rightarrow \infty} \int_{0}^{t}(N(\tau)-I(\tau)) d \tau=-N_{0}<0$. Thus, $\limsup \sup _{t \rightarrow \infty} \sup _{0 \leq \tau \leq t} N(t)=\lim _{t \longrightarrow \infty} N(t)=\infty$ as $t \longrightarrow \infty$ if $b>d+a$.

Case (ii): assume that $d<b \leq d+a$. Then, $\dot{N}(t) \leq a(N(t)-I(t)), \quad \forall t \in \mathbf{R}_{0+}, \quad$ from (5). Since limsup $\sup _{t \rightarrow \infty} \sup _{0 \leq \tau \leq t} N(t)<\infty$, it follows that $\lim _{t}$ $\longrightarrow \infty(I(t)-N(t))=\lim _{t \longrightarrow \infty}(S(t)+E(t)+R(t))$ $=0$. Thus, from Theorem 1 , one has that $\lim _{t} \longrightarrow \infty S$ $(t)=\lim _{t \rightarrow \infty} E(t)=\lim _{t \rightarrow \infty} R(t)=0$. One also gets from (2) that for any nontrivial solution of (1)-(4),

$$
\liminf _{t \longrightarrow \infty}\left[\sup _{0 \leq T<t}\left(\inf _{T \leq \tau \leq t} I(\tau)\right)\right] \geq c \liminf _{t \longrightarrow \infty}\left[\sup _{0 \leq T<t}\left(\inf _{T \leq \tau \leq t} \int_{0}^{t} e^{-(f+d)(t-\tau)} S(\tau) I(\tau) \mathrm{d} \tau\right)\right]>0,
$$

so that $(17)$ contradicts that $E(t) \longrightarrow 0$ as $t \longrightarrow \infty$. Hence, a contradiction occurs so that if $d<b \leq d+a$, then $N(t) \longrightarrow \infty$ as $t \longrightarrow \infty$.

As a result of the conclusions of Case (i) and Case (ii), $N(t) \longrightarrow \infty$ as $t \longrightarrow \infty$ if $b>d$. On the other hand, since $N(t) \longrightarrow \infty$ as $t \longrightarrow \infty$, then $S(t) \longrightarrow \infty$ as $t \longrightarrow \infty$. This also implies from (8) that $E(t) \longrightarrow \infty$ as $t \longrightarrow \infty$ or $I(t) \longrightarrow 0$ as $t \longrightarrow \infty$, but it implies also from (3) that $I(t) \longrightarrow \infty$ as $t \longrightarrow \infty$ since $T(t)$ is bounded. As a result, both $E(t) \longrightarrow \infty$ and $I(t) \longrightarrow \infty$ as $t \longrightarrow \infty$. Finally, $R(t) \longrightarrow \infty$ as $t \longrightarrow \infty$ from (9) since $I(t) \longrightarrow \infty$ as $t \longrightarrow \infty$. Thus, if the controls are bounded for all time and $b>d$, then all the subpopulations become unbounded as time tends to infinity. Property (ii) has been proved.

The subsequent result concerns the disease-free equilibrium point and sufficiency-type conditions for its local asymptotic stability and instability.

Theorem 3. Assume that the vaccination and treatment controls are generated via linear feedback as $V(t)=k_{V} S(t)$ and $T(t)=k_{T} I(t)$. The disease-free equilibrium point $x_{d f}^{*}=$ $\left(S_{d f}^{*}, E_{d f}^{*}, I_{d f}^{*}, R_{d f}^{*}\right)^{T}$ is locally asymptotically stable if and only if $S_{d f}^{*}<\left((f+d)\left(g+a+d+k_{T}\right) / c f\right)$ for $b \leq d$, which always holds in the case of asymptotic extinction (that is, if $b<d)$ and it is instable if $S_{d f}^{*}>\left((f+d)\left(g+a+d+k_{T}\right) / c f\right)$. If $b \leq d$, then disease-free equilibrium point is locally asymptotically stable if the transmission rate is small enough to satisfy $c<\left((f+d)\left(g+a+d+k_{T}\right)\left(b+k_{V}\right) / b f\right)$. Recent studies rely on the fact that the transmission rate of COVID 19 and, as a result, other respiratory diseases can be reduced through intervention actions such as the use of masks and limitation of numbers of attendees in public meetings or transportation [33].

Proof. Note that the Jacobian of the state trajectory solution around the disease-free equilibrium point is

$$
J_{d f}^{*}=\left[\begin{array}{cccc}
b-d-k_{V} & b & b-c S_{d f}^{*} & b \\
0 & -(f+d) & c S_{d f}^{*} & 0 \\
0 & f & -\left(g+a+d+k_{T}\right) & 0 \\
k_{V} & 0 & g+k_{T} & -d
\end{array}\right] .
$$

Then, the linearized subsystem of the exposed-infectious has the following characteristic equation and roots from (18):

$$
\begin{gathered}
s^{2}+\left(f+d+g+d+a+k_{T}\right) s+(f+d)\left(g+a+d+k_{T}\right)-c f S_{d f}^{*}=0, \\
s_{1,2}=\frac{-\left(f+d+g+d+a+k_{T}\right) \pm \sqrt{\left(f+d+g+d+a+k_{T}\right)^{2}+4\left(c f S_{d f}^{*}-(f+d)\left(g+a+d+k_{T}\right)\right)}}{2} .
\end{gathered}
$$


The following cases can tentatively occur from (20):

(1) The above roots are real and negative if and only if

$$
\begin{aligned}
& \frac{\left(f+d+g+d+a+k_{T}\right)^{2}-4(f+d)\left(g+a+d+k_{T}\right)}{4 c f} \\
& \leq S_{d f}^{*}<\frac{(f+d)\left(g+a+d+k_{T}\right)}{c f},
\end{aligned}
$$

and the disease-free equilibrium point is locally asymptotically stable subject to (21). These constraints also hold in the case of asymptotic extinction from Theorem 2 with $b \leq d$.

(2) The Jacobian matrix cannot have nonreal complex conjugate eigenvalues. Note that the characteristic roots are complex with negative real part if and only if

$$
\begin{aligned}
& \left(f+d+g+d+a+k_{T}\right)^{2} \\
& \quad-4\left((f+d)\left(g+a+d+k_{T}\right)-c f S_{d f}^{*}\right)<0
\end{aligned}
$$

that is, from (22), if and only if

$S_{d f}^{*}<\frac{4(f+d)\left(g+a+d+k_{T}\right)-\left(f+d+g+d+a+k_{T}\right)^{2}}{4 c f}$,

with (23) subject to

$$
\begin{aligned}
& \frac{\left(f+d+g+d+a+k_{T}\right)^{2}}{(f+d)\left(g+a+d+k_{T}\right)}=\frac{f+d}{g+a+d+k_{T}} \\
& +\frac{g+a+d+k_{T}}{f+d}+2<4,
\end{aligned}
$$

or, equivalently, provided that $\delta+\delta^{-1}<2$ with $\delta=\left(f+d / g+a+d+k_{T}\right)$, which is impossible.

(3) One root is positive if and only if $S_{d f}^{*}>((f+d)(g+$ $\left.a+d+k_{T}\right) / c f$ ) which implies local instability of the disease-free equilibrium point.

(4) One of the roots is zero if and only if $S_{d f}^{*}=((f+$ d) $\left.\left(g+a+d+k_{T}\right) / c f\right)$ which gives the critical stability susceptible value.
If $b<d$, the condition of local asymptotic stability of the disease-free equilibrium point always holds since $S_{d f}^{*}=N_{d f}^{*}=0$ from Theorem 2. On the other hand, if $b=d$, so that the disease-free equilibrium point is locally asymptotically stable without extinction, then, from Theorem 2 , the disease-free equilibrium is locally asymptotically stable if and only if the constraints (22) below hold:

$$
\begin{aligned}
\frac{b N_{d f}^{*}}{b+k_{V}} & =S_{d f}^{*} \leq \frac{b\left(N_{0}-a \int_{0}^{\infty} I(\tau) \mathrm{d} \tau\right)}{b+k_{V}} \leq \frac{b N_{0}}{b+k_{V}} \\
& <\frac{(f+d)\left(g+a+b+k_{T}\right)}{c f} .
\end{aligned}
$$

The stability depends only on the disease-free equilibrium point so the above constraint holds for a normalized model with $N_{0}=1$ if $c<\left((f+d)\left(g+a+d+k_{T}\right)\right.$ $\left.\left(b+k_{V}\right) / b f\right)$.

Remark 1. The condition of local asymptotic stability $S_{d f}^{*}<\left((f+d)\left(g+a+d+k_{T}\right) / c f\right)$ of the disease-free equilibrium point can be also characterized by the basic reproduction number $R_{b}$, which is the average number of primary contagions generated by each infectious individual, to be less than one. Let us define $R_{b}=\left(S_{d f}^{*} c f /(f+\right.$ d) $\left.\left(g+a+d+k_{T}\right)\right)$. Then, $\quad S_{d f}^{*}<((f+d)(g+a+d$ $\left.\left.+k_{T}\right) / c f\right)$ if and only if $R_{b}<1$. In the case of linear feedback vaccination of the form $V(t)=k_{V} S(t), S_{d f}^{*}=\left(b N_{d f}^{*}\right) / d+k_{V}$ if $b=d, a=0$ (i.e., there is no disease-related mortality), and the model is normalized to unity; then, $N_{d f}^{*}=N_{0}=1$ so that $R_{b}=\left(b c f /(f+d)\left(g+a+d+k_{T}\right)\left(d+k_{V}\right)\right)<1$ if and only if $c \leq c_{c}=\left((f+d)\left(g+a+d+k_{T}\right)\left(d+k_{V}\right) / b f\right)$, that is, if the transmission rate is under a critical value $c_{c}$. Note that such a critical value increases as the vaccination rate $k_{V}$ increases.

The subsequent result is concerned with the attainability and characterization of the endemic equilibrium point.

Theorem 4. Assume that that the vaccination and treatment controls are generated via linear feedback as $V(t)=k_{V} S(t)$ and $T(t)=k_{T} I(t), \forall t \in \mathbf{R}_{0+}$, and that $d<b<(g+a+d+$ $\left.k_{T}\right)(f+d) d /\left(\left(g+d+k_{T}\right)(f+d)+a d\right)$; then, there exists $a$ reachable, or attainable, endemic equilibrium point $x^{*}$ whose components are

$$
\begin{aligned}
S^{*} & =\frac{\left(g+a+d+k_{T}\right)(f+d)}{c f}, \\
E^{*} & =\frac{\left(d+k_{V}\right)(f+d)\left(g+a+d+k_{T}\right)^{2}(b-d)}{f c\left[d a(f+d-b)-(b-d)(f+d)\left(g+d+k_{T}\right)\right]}, \\
I^{*} & =\frac{\left(d+k_{V}\right)(f+d)\left(g+a+d+k_{T}\right)(b-d)}{c\left[d a(f+a-d)-(b-d)(f+d)\left(g+d+k_{T}\right)\right]},
\end{aligned}
$$




$$
R^{*}=\frac{\left(g+a+d+k_{T}\right)(f+d)}{d c}\left(\frac{\left(g+k_{T}\right)\left(d+k_{V}\right)(b-d)}{d c\left[d a(f+a-d)-(b-d)(f+d)\left(g+d+k_{T}\right)\right]}+\frac{k_{V}}{f}\right) .
$$

The equilibrium infectious/exposed and infectious/susceptible ratios are

$$
\begin{aligned}
& \frac{I^{*}}{E^{*}}=\frac{f}{g+a+d+k_{T}}, \\
& \frac{I^{*}}{S^{*}}=\frac{f\left(d+k_{V}\right)(b-d)}{d a(f+a-d)-(b-d)(f+d)\left(g+d+k_{T}\right)} .
\end{aligned}
$$

The endemic equilibrium point is not reachable if $d<b<$ $\left(\left(g+a+d+k_{T}\right)(f+d) d /\left(g+d+k_{T}\right)(f+d)+a d\right)$ does not hold.

Proof. By zeroing the time-derivative of (3) with $E^{*} \neq 0$ and $I^{*} \neq 0$, one gets

$$
I^{*}\left(1+\frac{k_{T}}{g+a+d}\right)=\frac{f}{g+a+d} E^{*}
$$

which is equivalent to (30) after simplification. Zeroing the time-derivative of (2) and comparing it to (30) yield

$$
c S^{*} I^{*}=(f+d) E^{*}=c S^{*} \frac{f E^{*}}{g+a+d+k_{T}},
$$

and canceling $E^{*}$ in both sides of the second equality leads to (26). Now, zeroing the time-derivative of (1) and using $N^{*}=$ $(a /(b-d)) I^{*}$ obtained from (5) with zero time-derivative of the total population yield

$$
\left(\frac{b a}{b-d}-c S^{*}\right) I^{*}=\left(d+k_{V}\right) S^{*}
$$

provided that $b>d$ and $S^{*}<(b a / c(b-d))$ which according to the equilibrium susceptible subpopulation in (26) is equivalent to

$$
\frac{b}{b-d}>\xi=\frac{\left(g+a+d+k_{T}\right)(f+d)}{a f},
$$

which after rearranging and simplifying terms yields

$$
b<\frac{\xi d}{\xi-1}=\frac{\left(g+a+d+k_{T}\right)(f+d) d}{\left(g+d+k_{T}\right)(f+d)+a d} .
$$

The case $b=d$ and $S^{*}<(b a / c(b-d))$ is excluded since the total population is nonnegative and bounded from Theorem 1. It has been proved that if $d<b<\bar{b}=((g+a+$ $\left.\left.d+k_{T}\right)(f+d) d /\left(\left(g+d+k_{T}\right)(f+d)+a d\right)\right)$ fails, then the endemic equilibrium point is not reachable since the infectious subpopulation is either negative or unbounded. Now, one replaces (26) into (34) subject to the above constraint to yield

$$
\begin{aligned}
I^{*} & =\frac{d+k_{V}}{(b a /(b-d))-c S^{*}} S^{*} \\
& =\frac{\left(d+k_{V}\right)(b-d)\left(g+a+d+k_{T}\right)(f+d)}{f b a-(b-d)\left(g+a+d+k_{T}\right)(f+d)},
\end{aligned}
$$

whose denominator is positive if $d<b<\bar{b}$ which is equivalent to $S^{*}<(b a /(c(b-d)))$ and which yields (28) after canceling terms of distinct sign in the denominator. On the other hand, (31) follows from (26) into (37) and (27) follows from (28) and (30). Finally, by zeroing the time-derivative in (4), one gets

$$
R^{*}=\frac{\left(g+k_{T}\right) I^{*}+k_{V} S^{*}}{d},
$$

which yields (29) after using (26) and (28).

Note that if $b<d$, then the endemic equilibrium point is unreachable (or unattainable) since it has negative components. If $b>d$, then the exposed and infectious components are positive so that the point is reachable (or attainable). Since the endemic equilibrium is unattainable if $b \leq d$ and the disease-free equilibrium point is guaranteed to be locally asymptotically stable if $c<((f+d)(g+a+d+$ $\left.\left.k_{T}\right)\left(b+k_{V}\right)\right) / b f$ and $b \leq d$, it follows from Theorems 3 and 4 that the local asymptotic stability of the disease-free equilibrium point is also global if $c<((f+d)(g+a+$ $\left.\left.d+k_{T}\right)\left(b+k_{V}\right)\right) / b f$ and $b \leq d$; the next direct result is established.

Corollary 2. Assume that that the vaccination and treatment controls are generated via linear feedback as $V(t)=k_{V} S(t)$ and $T(t)=k_{T} I(t), \forall t \in \mathbf{R}_{0+}$, and that $b \leq d$. Then, the disease-free equilibrium point is guaranteed to be globally asymptotically stable if $b \leq d$ and $c<((f+d)(g+a+d$ $\left.\left.+k_{T}\right)\left(b+k_{V}\right)\right) / b f$.

\section{A Special Epidemic Model with Habitat and Flux Population-Dependent Recruitment and Its Approximate Output- Reachability Properties}

Note that the output-reachability concept is basically related to the property of the selected output to reach a prescribed arbitrary value at a certain predefined time instant by the application of a control signal. It is known that it is not possible for all the systems to drive the output to a desired level in a defined amount of time. Therefore, this section determines whether the considered epidemic model has this property or not.

The output-reachability is of interest for epidemic systems since the desired values of the subpopulations can be set easily in order to eradicate the infection if the infectious subpopulation evolution is defined as the "output." With these mentioned targeted values, the control signal can be calculated by well-known techniques to achieve the desired 
objective within a certain period of time. Thus, this approach directly provides the amount of control action needed, which in turn allows estimating the size of vaccination, treatment, or other counteraction measures needed. This is the main issue of the paper which is novel in the study of epidemic systems.

Now, this section studies a special model which relates the global net recruitment to the birth rate and outwards and inwards population flux rates together. Later on, its approximate linear output-reachability properties are investigated based on an ad hoc point-to-point linearization of the state trajectory solution. The output is necessarily of a lower dimensionality than that of the whole state since the epidemic model is neither (state) controllable to the origin, even if the disease-free equilibrium point is globally asymptotically stable, nor reachable. A particular "output" of interest can be defined as the infectious subpopulation. Subsequently, a control design is proposed to track approximately successive sampled values of the output fixed as objectives of the output trajectory suited profiles. The distribution of the samples can be organized with constant or with adaptive sampling rates between each two consecutive samples.

4.1. The Special Model with Recruitment Description. A special form of the recruitment is now discussed which agrees with what intuition dictates in the sense that the recruitment depends on the total population and eventual inward/outward population rates. It is assumed that the recruitment tends to an asymptotic limit as the illness, due to technical healthcare decisions such as forced quarantines or authority emergency policies, evolves which eventually depends on the initial value of the population model parameters and, in particular, on the disease mortality rate $a$. Assume the following modification of the model (1)-(4) with population recruitment given by $\lambda(t)=b N(t)+\widetilde{\lambda}(t)$, $\forall t \in \mathbf{R}_{0+}$, describing a part being proportional to the total population and another one describing a recruitment generated by rates of flux of inward and outward population, assumed to be subject to $\lambda(t) \longrightarrow \lambda^{*}, \widetilde{\lambda}(t) \longrightarrow \vec{\lambda}^{*}\left(<\lambda^{*}\right)$ as $t \longrightarrow \infty$ with, eventually, $\lambda^{*}=\lambda^{*}\left(N_{0}, p\right)$, with $p$ being the parameter vector of the model, so that the total population also converges asymptotically $N(t) \longrightarrow\left(\lambda^{*}-\vec{\lambda}^{*}\right) / b$ as $t \longrightarrow \infty$. Assume that linear feedback vaccination and treatment controls $V(t)=k_{V} S(t)$ and $T(t)=k_{T} I(t)$, $\forall t \in \mathbf{R}_{0+}$, with $T(t) \longrightarrow 0$ as $t \longrightarrow \infty$ :

$$
\dot{S}(t)=\lambda(t)-\left(d+k_{V}\right) S(t)-c S(t) I(t), \quad \forall t \in \mathbf{R}_{0+},
$$

which leads to

$$
\dot{N}(t)=\lambda(t)-d N(t)-a I(t), \quad \forall t \in \mathbf{R}_{0+} .
$$

Then, the disease-free equilibrium point for $b \leq d$ is defined by $E_{d f}^{*}=I_{d f}^{*}=0$, and

$$
\begin{aligned}
& S_{d f}^{*}=\frac{\lambda^{*}-\tilde{\lambda}^{*}}{d+k_{V}}, \\
& R_{d f}^{*}=\frac{k_{V}}{d} S_{d f}^{*}=\frac{k_{V}\left(\lambda^{*}-\tilde{\lambda}^{*}\right)}{d\left(d+k_{V}\right)} .
\end{aligned}
$$

Note that $R_{d f}^{*-1}=\left(d /\left(\lambda^{*}-\tilde{\lambda}^{*}\right)\right)\left(1+\left(d / k_{V}\right)\right)$ so that it follows that $S_{d f}^{*}$ decreases and $R_{d f}^{*}$ increases as $k_{V}$ increases. Moreover,

$$
\begin{aligned}
& N(t)=e^{-\mathrm{d} t} N_{0}+\int_{0}^{t} e^{-\mathrm{d}(t-\tau)}(\lambda(\tau)-a I(\tau)) \mathrm{d} \tau, \\
& \frac{1}{d} \liminf _{t}\left(\inf _{0 \leq \tau \leq t}(\lambda(\tau)-a I(\tau))\right) \leq \lim _{t \rightarrow \infty} N(t)=\frac{\lambda^{*}-\tilde{\lambda}^{*}}{b} \\
& =\lim _{t \rightarrow \infty}\left(e^{-\mathrm{d} t} \int_{0}^{t} e^{\mathrm{d} \tau}(\lambda(\tau)-a I(\tau)) \mathrm{d} \tau\right) \\
& \leq \frac{1}{d} \limsup _{t \longrightarrow \infty}\left(\sup _{0 \leq \tau \leq t}(\lambda(\tau)-a I(\tau))\right) .
\end{aligned}
$$

The Jacobian matrix of the linearized state trajectory solution around $x(t)=(S(t), E(t), I(t), R(t))^{T}$ under eventual time-varying control gains $k_{V}(t) \longrightarrow k_{V}^{*}$, $k_{T}(t) \longrightarrow k_{T}^{*}$ (as $t \longrightarrow \infty$ ) at any time instant $t$ becomes

$$
J(x(t))=\left[\begin{array}{cccc}
b-d-k_{V}(t) & b & b-c S(t) & b \\
0 & -(f+d) & c S(t) & 0 \\
-c I(t) & f+c I(t) & -\left(g+a+d+k_{T}(t)\right) & 0 \\
k_{V}(t) & 0 & g+k_{T}(t) & -d
\end{array}\right] .
$$

As time tends to infinity, and provided that the infectious subpopulation asymptotically converges to zero, the above Jacobian matrix tends to that of the disease-free equilibrium point; that is,

$$
\lim _{t \longrightarrow \infty} J(x(t))=\lim _{I(t) \longrightarrow 0} J(x(t))=J_{d f}^{*} .
$$

4.2. Approximate Reachability Issues. The linearized trajectory solution can be approximated from a linearized differential system $\dot{x}(t)=J(x(t)) x(t)$, by assuming that the state is constant in any interval $[t, t+\Delta t)$, as follows. Assume that the fundamental matrix of the above linearized differential system is the solution of $\dot{\psi}(t, 0)=J(t, x(t)) \psi(t, 0)$ with $\psi(0,0)=I_{4}$ (the four-dimensional identity matrix); then, for a sufficiently small $\Delta t$, 


$$
\begin{aligned}
x(t+\Delta t)= & \psi(t, 0) x(t) \\
& +\int_{0}^{\Delta t} \psi(\tau, t) B u(t+\tau) \mathrm{d} \tau \approx e^{A(S(t), I(t)) \Delta t} x(t) \\
& +\int_{0}^{\Delta t} e^{A(S(t), I(t))(\Delta t-\tau)} B u(t+\tau) \mathrm{d} \tau, \quad \forall t \in \mathbf{R}_{0+},
\end{aligned}
$$

such that the approximated above expression is got by keeping the model state constant on the time interval $[t, t+\Delta t]$, where

$$
\begin{aligned}
A(S(t), I(t)) & =\left[\begin{array}{cccc}
b-d & b & b-c S(t) & b \\
0 & -(f+d) & c S(t) & 0 \\
-c I(t) & f+c I(t) & -(g+a+d) & 0 \\
0 & 0 & g & -d
\end{array}\right], \\
B & =\left[\begin{array}{cc}
-1 & 0 \\
0 & 0 \\
0 & -1 \\
1 & 1
\end{array}\right], \\
u(t) & =\left[\begin{array}{c}
V(t) \\
T(t)
\end{array}\right],
\end{aligned}
$$

$\forall t \in \mathbf{R}_{0+}$. Under linear control feedback, (47) can be particularized with the particular control matrix and control input given by

$$
\begin{aligned}
& B(t)=\left[\begin{array}{cc}
-V(t) & 0 \\
0 & 0 \\
0 & -I(t) \\
V(t) & I(t)
\end{array}\right], \\
& u(t)=\left[\begin{array}{c}
k_{V}(t) \\
k_{I}(t)
\end{array}\right],
\end{aligned}
$$

so that, for sufficiently small $n \Delta t$ compared to the disease evolution profile, one has in a similar way to (46):

$$
\begin{aligned}
x(t+n \Delta t) \approx & \left(\prod_{i=0}^{n-1}\left[e^{A(S(t+i \Delta t), I(t+i \Delta t)) \Delta t}\right]\right) x(t) \\
& +\sum_{i=0}^{n-1} \prod_{j=i}^{n-1}\left[e^{A(S(t+(n-1-j) \Delta t), I(t+(n-1-j) \Delta t)) \Delta t}\right] \\
& \cdot \int_{0}^{\Delta t} e^{A(S(t+i \Delta t), I(t+i \Delta t))(\Delta t-\tau)} B u(t+i \Delta t+\tau) \mathrm{d} \tau .
\end{aligned}
$$

It is easy to see that the model, as is the common case in all the epidemic models, is not reachable in the sense that it is not possible for the whole state (that is, for all the subpopulations) to target arbitrary prefixed nonnegative values at any prescribed finite time as the following simple and direct result is established.

Theorem 5. The approximated linearized model (46) and (47) about the disease-free equilibrium point is not reachable.

Proof. Assume that three subpopulations are reachable from the disease-free equilibrium point. With no loss in generality, assume that in any given finite time $T_{a}>0$ there exist a control $u^{*}(t)=\left(V^{*}(t), I^{*}(t)\right)^{T}$ on $\left[0, T_{a}\right)$ which is able to transfer $S_{0}=S_{d f}^{*}, E_{0}=E_{d f}^{*}=0$ and $I_{0}=I_{d f}^{*}=0$ at $t_{0}=0$ to prescribed values $S\left(T_{a}\right)=S^{*}\left(T_{a}\right) \geq 0, E\left(T^{*}\right)=$ $E^{*}\left(T_{a}\right) \geq 0$, and $I\left(T_{a}\right)=I^{*}\left(T_{a}\right) \geq 0$ at $t=T_{a}$ such that $\left(S^{*}\left(T_{a}\right), E^{*}\left(T_{a}\right), I^{*}\left(T_{a}\right)\right)^{T} \neq\left(S_{d f}^{*}, 0,0\right)^{T}$. From $(42), \quad N$ $\left(T_{a}\right)=e^{-\mathrm{d} T_{a}\left(S_{d f}^{*}+R_{d f}^{*}\right)+\int_{0}^{T_{a}} e^{-} \mathrm{d}\left(T_{a}-\tau\right)}(\lambda(\tau)-a I(\tau)) \mathrm{d} \tau$ and then

$$
R\left(T_{a}\right)=e^{-\mathrm{d} T_{a}}\left(S_{d f}^{*}+R_{d f}^{*}\right)+\int_{0}^{T_{a}} e^{-\mathrm{d}\left(T_{a}-\tau\right)}(\lambda(\tau)-a I(\tau)) \mathrm{d} \tau-S^{*}\left(T_{a}\right)-E^{*}\left(T_{a}\right)-I^{*}\left(T_{a}\right)
$$

Therefore, $R^{*}\left(T_{a}\right)$ cannot be distinct from the above value $R\left(T_{a}\right)$, and then it cannot be fixed arbitrarily. Thus, the linearized system is not reachable.

Remark 2. Note that Theorem 5 has not considered the constraints on the vaccination and treatment controls, but it is obvious that under these additional constraints the system is not reachable either. The basic reason is that the whole population is independent of the controls so that at least one of the subpopulations is forced by the other ones. In addition, there is no control feasible on the exposed subpopulation. Then, the reachability lack in epidemic models is rather structural and linked to the nature of epidemic diseases, since the controls are compensatory in the recovered subpopulation since they are incorporated as recovered subpopulation the corresponding deleted numbers of the susceptible and/or infected.

To overcome the loss of reachability of the whole state, it is possible to define some measurable outputs having less components than the state, for instance, with three (or less) of the subpopulations or three (or less) linear combinations of the subpopulations. In this way, the system might be 
output reachable in the sense that the output itself (rather than the whole state) is reachable. The subsequent developments go in that direction.

Consider the control $u(t+i \Delta t+\tau)$ for $i=0,1, \ldots, n-1$ and any given $t \in \mathbf{R}_{0+}$ being generated from an auxiliary control $v(t)$ to be then fixed, which generates the control $u:[t, t+(n-1) \Delta t) \longrightarrow \mathbf{R}_{0+}^{2}$ of the form $\{u(t+i \Delta t-\tau) ; \tau \in[0, \Delta t)\}_{i=0}^{n-1}$ as follows:

$$
u(t+i \Delta t-\tau)=B^{T} e^{A(S(t+i \Delta t), I(t+i \Delta t))(\Delta t-\tau)} C^{T} v(t), \quad \tau \in[0, \Delta t) i=0,1, \ldots, n-1
$$

on the time interval $[t, t+(n-1) \Delta t)$ to target an approximate prefixed tentative output point $y^{*}=y\left(t+T_{a}\right)=$ $C x\left(t+T_{a}\right)$ at time instant $t+T_{a}=t+n \Delta t$ from an initial time instant $t$, for some defined output matrix $C \in \mathbf{R}^{p \times 4}$ with some $1 \leq p<4$, since the whole system cannot be reachable (see Theorem 1), but it can be potentially output-reachable for some appropriate choice of the output matrix. The output-reachability objective at $\left(t+T_{a}\right)$ driven by the auxiliary control input $v(t)$ is established as follows:

$$
\begin{aligned}
& y^{*}-C\left(\prod_{i=0}^{n-1}\left[e^{A(S(t+i \Delta t), I(t+i \Delta t)) \Delta t}\right]\right) x(t) \\
& \cong C \sum_{i=0}^{n-1} \prod_{j=i}^{n-1}\left(\left[e^{A(S(t+(n-1-j) \Delta t), I(t+(n-1-j) \Delta t)) \Delta t}\right] \times\left(\int_{0}^{\Delta t} \begin{array}{c}
e^{A(S(t+i \Delta t), I(t+i \Delta t))(\Delta t-\tau)} \\
B B^{T} e^{A^{T}(S(t+i \Delta t), I(t+i \Delta t))(\Delta t-\tau)} \mathrm{d} \tau
\end{array}\right)\left(\prod_{j=i}^{n-1}\left[e^{A(S(t+(n-1-j) \Delta t), I(t+(n-1-j) \Delta t)) \Delta t}\right]\right) C^{T} v(t) .\right.
\end{aligned}
$$

The following definition is useful for addressing the subsequent result.

Definition 1. The linearized model (46) and (47) with measurable output $y(t)=C x(t)$ for some full rank output matrix $C \in \mathbf{R}^{p \times 4}(1 \leq p<4)$ is said to be $(n, \Delta t)$-step-to-step output-reachable through unconstrained control on $\left[t, t+T_{a}\right)$, with $T_{a}=n \Delta t$, for a given $t \in \mathbf{R}_{0+}$ if there is some control $u^{*}(t)=\left(V^{*}(t), T^{*}(t)\right)^{T}$ such that the state $x(t)$ at the time instant $t$ leads to a prefixed linearized output $y\left(t+T_{a}\right)=C x\left(t+T_{a}\right)=y^{*}$.

For instance, if the targeted output is the infectious subpopulation, then $C=c^{T}=(0,0,1,0)$. Note that Definition 1 does not require that the control function be constrained and that the targeted output be nonnegative. As a result, its practical applicability to the epidemic model will require some additional considerations to be discussed later on. The next result is related to the use of Definition 1 and considers also the case of control boundedness to keep the nonnegativity of the state trajectory solution of Definition 1.

Theorem 6. Assume that the linearized model (46) and (47) is $(n, \Delta t)$-step-to-step output-reachable through unconstrained control on $\left[t, t+T_{a}\right)$ with $T_{a}=n \Delta t$ for a measurable output defined by $y(t)=C x(t)$ through some full rank output matrix $C \in \mathbf{R}^{p \times 4}$ for some $1 \leq p<4$. Then, the following properties hold:

(i) The $(n, \Delta t)$-step-to-step output-controllability Gramian on $\left[t, t+T_{a}\right]$ defined by

$$
\begin{aligned}
\mathbf{C}\left(t, t+T_{a}\right)= & C \sum_{i=0}^{n-1}\left(\prod_{j=i}^{n-1}\left[e^{A(S(t+(n-1-j) \Delta t), I(t+(n-1-j) \Delta t)) \Delta t}\right] \times\left(\int_{0}^{\Delta t} e^{A(S(t+i \Delta t), I(t+i \Delta t))(\Delta t-\tau)} B B^{T} e^{A^{T}(S(t+i \Delta t), I(t+i \Delta t))(\Delta t-\tau)} \mathrm{d} \tau\right)\right. \\
& \left.\times\left(\prod_{j=i}^{n-1}\left[e^{A(S(t+(n-1-j) \Delta t), I(t+(n-1-j) \Delta t)) \Delta t}\right]\right)\right) C^{T}
\end{aligned}
$$




$$
\begin{aligned}
& u^{*}(t+i \Delta t-\tau)=\left[\begin{array}{c}
V^{*}(t+i \Delta t-\tau) \\
T^{*}(t+i \Delta t-\tau)
\end{array}\right] \\
&=B^{T} e^{A(S(t+i \Delta t), I(t+i \Delta t))(\Delta t-\tau)} C^{T} \mathbf{C}^{-1}\left(t, t+T_{a}\right)\left(y^{*}-C\left(\prod_{i=0}^{n-1}\left[e^{A(S(t+i \Delta t), I(t+i \Delta t)) \Delta t}\right]\right) x(t)\right), \\
& \tau \in[0, \Delta t) i=0,1, \ldots, n-1,
\end{aligned}
$$

leads to and approximated targeted output value $y\left(t+T_{a}\right)=y^{*}+o\left(T_{a}\right)$ so that $\lim _{\Delta t \rightarrow 0}\left(y\left(t+T_{a}\right)-y^{*}\right)=0$ for a prefixed $n$ and any given $x(t)$.

(iii) Assume that the linear controls of Property (ii) are subject to the nonnegativity boundedness constraints

$$
\begin{aligned}
\bar{u}^{*}(t+i \Delta t-\tau) & =\left[\begin{array}{l}
\bar{V}^{*}(t+i \Delta t-\tau) \\
\bar{T}^{*}(t+i \Delta t-\tau)
\end{array}\right] \\
& =B^{T} e^{A(S(t+i \Delta t), I(t+i \Delta t))(\Delta t-\tau)} C^{T} \mathbf{C}^{-1}\left(t, t+T_{a}\right)\left(y^{*}-C\left(\prod_{i=0}^{n-1}\left[e^{A(S(t+i \Delta t), I(t+i \Delta t)) \Delta t}\right]\right) x(t)\right), \quad \tau \in[0, \Delta t) i=0,1, \ldots, n-1,
\end{aligned}
$$

$V^{*}(t+i \Delta t-\tau)=\left\{\begin{array}{ll}\bar{V}(t+i \Delta t-\tau), & \text { if } 0<\bar{V}(t+i \Delta t-\tau) \leq b N(t+i \Delta t-\tau), \\ 0, & \text { if } \bar{V}(t+i \Delta t-\tau) \leq 0, \\ b N(t+i \Delta t-\tau), & \text { if } \bar{V}(t+i \Delta t-\tau)>b N(t+i \Delta t-\tau),\end{array} \quad \tau \in[0, \Delta t) i=0,1, \ldots, n-1\right.$,

$$
T^{*}(t+i \Delta t-\tau)=\left\{\begin{array}{ll}
\bar{T}(t+i \Delta t-\tau), & \text { if } 0<\bar{T}(t+i \Delta t-\tau) \leq f E(t+i \Delta t-\tau), \\
0, & \text { if } \bar{T}(t+i \Delta t-\tau) \leq 0, \\
f E(t+i \Delta t-\tau), & \text { if } \bar{T}(t+i \Delta t-\tau)>f E(t+i \Delta t-\tau),
\end{array} \quad \tau \in[0, \Delta t), i=0,1, \ldots, n-1\right.
$$

where

$$
\begin{aligned}
\bar{V}(t+i \Delta t-\tau)=e_{1}^{T} u^{*}(t+i \Delta t-\tau), & \tau \in[0, \Delta t) i=0,1, \ldots, n-1, \\
\bar{T}(t+i \Delta t-\tau)=e_{2}^{T} u^{*}(t+i \Delta t-\tau), & \tau \in[0, \Delta t) i=0,1, \ldots, n-1, \\
u^{*}(t+i \Delta t-\tau)=\left[\begin{array}{c}
V^{*}(t+i \Delta t-\tau) \\
T^{*}(t+i \Delta t-\tau)
\end{array}\right], & \tau \in[0, \Delta t) i=0,1, \ldots, n-1,
\end{aligned}
$$

where $e_{i}$ is the $i$-th canonical unity vector of $\mathbf{R}^{p}$. The output targeting error related to $y^{*}\left(T_{a}\right)$ is

$$
\left\|\widetilde{y}\left(T_{a}\right)\right\|=\left\|C \sum_{i=0}^{n-1} \prod_{j=i}^{n-1}\left[e^{A(S(t+(n-1-j) \Delta t), I(t+(n-1-j) \Delta t)) \Delta t}\right] \int_{0}^{\Delta t} e^{A(S(t+i \Delta t), I(t+i \Delta t))(\Delta t-\tau)} B\left[\begin{array}{c}
\bar{V}(t)-V^{*}(t) \\
\bar{T}(t)-T^{*}(t)
\end{array}\right] \mathrm{d} \tau\right\|+o\left(T_{a}\right) .
$$

Proof. From (51) and Definition 1, the mappings $v(t) \longrightarrow f(u(t+i \Delta t-\tau) ; \tau \in[0, \Delta t), i=0,1, \ldots, n-1)$ and $(u(t+i \Delta t-\tau) ; \tau \in[0, \Delta t), i=0,1, \ldots, n-1) \longrightarrow^{g} t y n(t+$ $\left.T_{a}\right)$ ) are injective and surjective, respectively. Thus, the composite mapping $g \circ f$ is bijective for an auxiliary control: 


$$
v(t)=\mathbf{C}^{-1}\left(t, t+T_{a}\right)\left(y^{*}-C\left(\prod_{i=0}^{n-1}\left[e^{A(S(t+i \Delta t), I(t+i \Delta t)) \Delta t}\right]\right) x(t)\right), \quad t \in \mathbf{R}_{0+} \cdot
$$

Since such a bijective linear mapping $g \circ f$ is represented by the output-controllability Gramian $\mathbf{C}\left(t, t+T_{a}\right)$ defined in (53) with a square real $p$-matrix, such a matrix is full rank and then nonsingular. Property (i) has been proved. Property (iii) follows from Property (ii) and the control constraints $0 \leq V(t) \leq b N(t)$ and $0 \leq T(t) \leq f E(t), \forall t \in \mathbf{R}_{0+}$, of Theorem 1 .

4.3. Successive Sampled Output Targeting Procedure. The above result can be adapted to a successive output targeting of tentatively prefixed values on a time interval $\left[t, t+\ell T_{a}\right]$ with $T_{a}=n \Delta t$ in order to track tentatively prescribed linearized output samples $\left\{y^{*}\left(j T_{a}\right)\right\}_{j=1}^{\ell}$. There are several error sources such as the linearization of the solution trajectory and the use of constant $\Delta t$-interval values of the state. The errors become more relevant as the whole evaluation interval $\ell T_{a}$ and the sampling interval length $\Delta t$ increase. The basic equations to perform the output tracking are

$$
\begin{aligned}
& \bar{u}^{*}\left(t+j T_{a}+i \Delta t-\tau\right)=\left[\begin{array}{l}
\bar{V}^{*}\left(t+j T_{a}+i \Delta t-\tau\right) \\
\bar{T}^{*}\left(t+j T_{a}+i \Delta t-\tau\right)
\end{array}\right] \\
& =B^{T} e^{A\left(s\left(t+j T_{a}+i \Delta t\right), I\left(t++j T_{a}+i \Delta t\right)\right)(\Delta t-\tau)} C^{T} \mathbf{C}^{-1}\left(t+j T_{a}, t+(j+1) T_{a}\right) \\
& \quad \times\left(y^{*}-C\left(\prod_{i=0}^{n-1}\left[e^{A\left(s\left(t+j T_{a}+i \Delta t\right), I\left(t+j T_{a}+i \Delta t\right)\right) \Delta t}\right]\right) x\left(t+j T_{a}\right)\right), \quad \tau \in[0, \Delta t) i=0,1, \ldots, n-1 j=0,1, \ldots, \ell-1, \\
& V^{*}\left(t+j T_{a}+i \Delta t-\tau\right)=\left\{\begin{array}{ll}
\bar{V}\left(t+j T_{a}+i \Delta t-\tau\right), & \text { if } 0<\bar{V}\left(t+j T_{a}+i \Delta t-\tau\right) \leq b N\left(t+j T_{a}+i \Delta t-\tau\right), \\
0, & \text { if } \bar{V}\left(t+j T_{a}+i \Delta t-\tau\right) \leq 0, \\
b N\left(t+j T_{a}+i \Delta t-\tau\right), & \text { if } \bar{V}\left(t+j T_{a}+i \Delta t-\tau\right)>b N\left(t+j T_{a}+i \Delta t-\tau\right),
\end{array} \quad \tau \in, \Delta t\right) i=0,1, \ldots, n-1 j=0,1, \ldots, \ell-1, \\
& T^{*}\left(t+j T_{a}+i \Delta t-\tau\right)= \begin{cases}\bar{T}\left(t+j T_{a}+i \Delta t-\tau\right), & \text { if } 0<\bar{T}\left(t+j T_{a}+i \Delta t-\tau\right) \leq f E\left(t+j T_{a}+i \Delta t-\tau\right), \\
0, & \text { if } \bar{T}\left(t+j T_{a}+i \Delta t-\tau\right) \leq 0, \\
f E\left(t+j T_{a}+i \Delta t-\tau\right), & \text { if } \bar{T}\left(t+j T_{a}+i \Delta t-\tau\right)>f E\left(t+j T_{a}+i \Delta t-\tau\right),\end{cases}
\end{aligned}
$$

where

$$
\begin{aligned}
& \bar{V}\left(t+j T_{a}+i \Delta t-\tau\right)=e_{1}^{T} u^{*}\left(t+j T_{a}+i \Delta t-\tau\right), \quad \tau \in[0, \Delta t) i=0,1, \ldots, n-1 j=0,1, \ldots, \ell-1, \\
& \bar{T}\left(t+j T_{a}+i \Delta t-\tau\right)=e_{2}^{T} u^{*}\left(t+j T_{a}+i \Delta t-\tau\right), \quad \tau \in[0, \Delta t) i=0,1, \ldots, n-1 j=0,1, \ldots, \ell-1, \\
& u^{*}\left(t+j T_{a}+i \Delta t-\tau\right)=\left[\begin{array}{c}
V^{*}\left(t+j T_{a}+i \Delta t-\tau\right) \\
T^{*}\left(t+j T_{a}+i \Delta t-\tau\right)
\end{array}\right], \quad \tau \in[0, \Delta t) i=0,1, \ldots, n-1 j=0,1, \ldots, \ell-1,
\end{aligned}
$$

where $e_{i}$ is the $i$-th canonical unity vector of $\mathbf{R}^{p}$. The output targeting errors related to $y^{*}\left(j T_{a}\right)$ are

$$
\begin{aligned}
\left\|\tilde{y}\left(j T_{a}\right)\right\|= & \left\|C \sum_{i=0}^{n-1} \prod_{j=i}^{n-1}\left[e^{A\left(s\left(t+j T_{a}+(n-1-j) \Delta t\right), I\left(t+j T_{a}+(n-1-j) \Delta t\right)\right) \Delta t}\right] \times \int_{0}^{\Delta t} e^{A\left(s\left(t+j T_{a}+i \Delta t\right), I\left(t+j T_{a}+i \Delta t\right)\right)(\Delta t-\tau)} B\left[\begin{array}{c}
\bar{V}\left(t+j T_{a}\right)-V^{*}\left(t+j T_{a}\right) \\
T\left(t+j T_{a}\right)-T^{*}\left(t+j T_{a}\right)
\end{array}\right] \mathrm{d} \tau\right\| \\
& +o\left(\ell T_{a}\right), \quad \tau \in[0, \Delta t) i=0,1, \ldots, n-1 j=0,1, \ldots, \ell-1 .
\end{aligned}
$$


It is well-known that, in the general control context, the sampling rates can be adapted to the signal variations to improve the sampling efficiency by decreasing or increasing it as the signal varies faster or slower, respectively, or to decrease the influence of the relative measuring errors towards the computed results in computation problems based on algebraic relations. Adaptive sampling can improve the results by decreasing (respectively, increasing) the sampling intervals, which now become time-dependent, as the output time-derivative between consecutive examples increases (respectively, decreases). In the past, several adaptive sampling laws and some design techniques for nonuniform sampling have been proposed in the literature for different control and numerical problems. See, for instance, [23-30] and some of the references therein. The adaptation can be performed on the largest sampling period $T_{a}$ or on the smaller one $\Delta t$, now becoming with varying values $T_{a j}$ and $\Delta t_{i}$, respectively. The following updating laws can be used.

4.3.1. Adaptation of the Smaller Sampling Period. Note that the total operation interval $\left[t, t+\sum_{j=1}^{\ell} T_{a j}\right]$ is defined by the large and small sampling periods:

$$
\begin{aligned}
T_{a j}= & T_{a, j-1}+\sum_{i=1}^{n} \Delta t_{a i}, j=1,2, \ldots, \ell, \text { with } T_{a 0}=t, \\
\Delta t_{j i}= & \max \left(\Delta t_{m}, \frac{K_{1}}{K_{2}+K_{3}\left\|\dot{y}\left(T_{a j}+\Delta t_{j, i-1}\right)-\dot{y}\left(T_{a j}\right)\right\|}\right) \\
& \in\left[T_{a j}, T_{a, j+1}\right), \quad j=0,1, \ldots, \ell-1,
\end{aligned}
$$

with design constants $K_{i}>0, i=1,2,3$, which satisfy $\left(K_{1} / K_{2}\right)=\Delta t_{M}$, where $\Delta t_{m}<\Delta t_{M}<\infty$ is a maximum prescribed value for the small sampling period of the sequence $\left\{\Delta t_{j i}\right\}_{j=0, i=0}^{j=\ell, i=n}$ and $\Delta t_{m}>0$ is its minimum prescribed value. The successive sampling instants are given by the sequence $\left\{t_{i}\right\}_{i=0}^{\ell n}$, where the initial sampling time is the initial testing time; that is, $t_{0}=t$, and

$$
t_{i}=t+\sum_{j=0}^{[i / n]} T_{a j}+\sum_{k=0}^{i-[i / n] n} \Delta t_{[i / n] k}, \quad i=1,2, \ldots, \ell n
$$

by using (64), which leads to

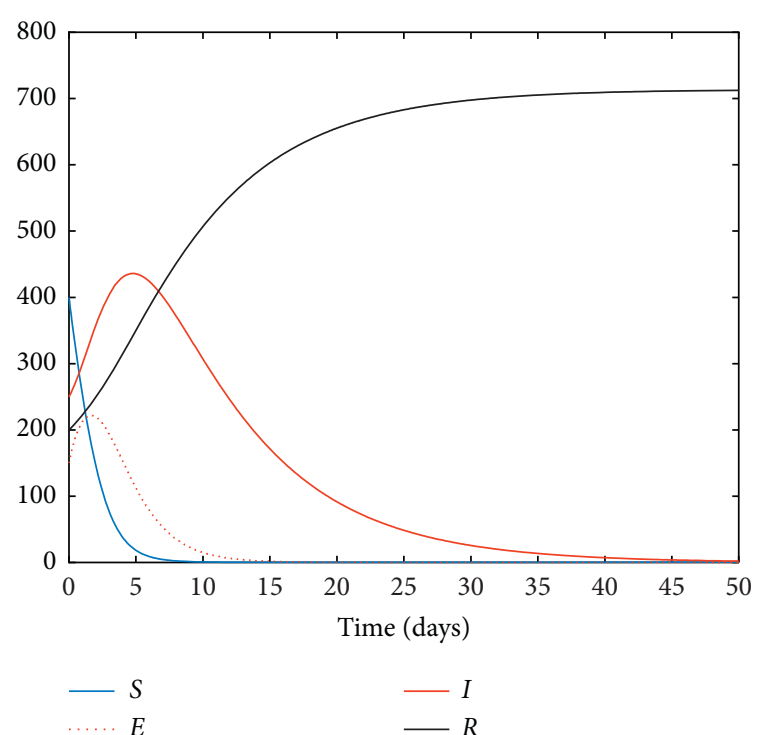

FIGURE 1: Evolution of the populations in the absence of external control actions.

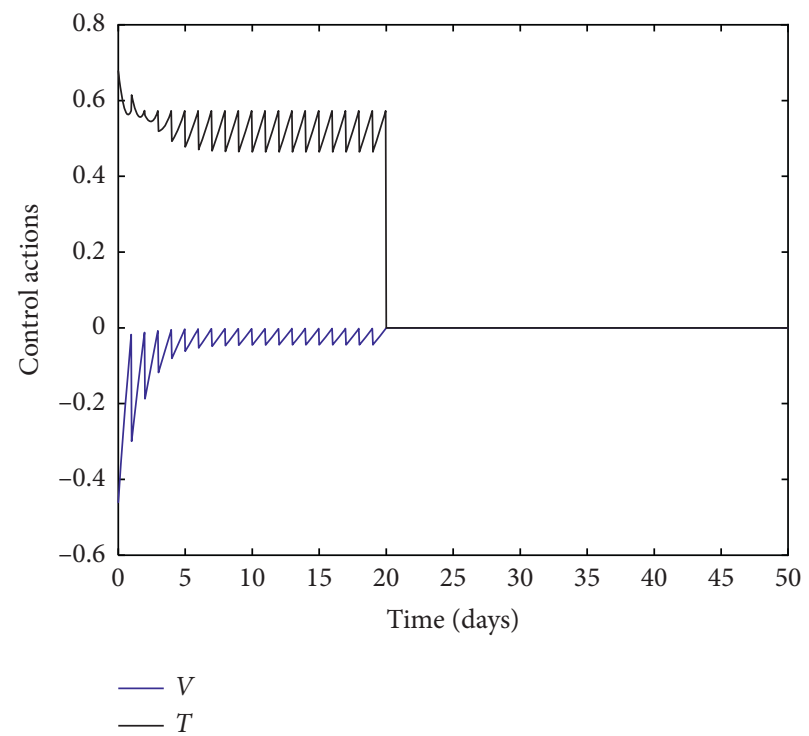

FIgURE 2: Vaccination and treatment actions calculated from (54). 


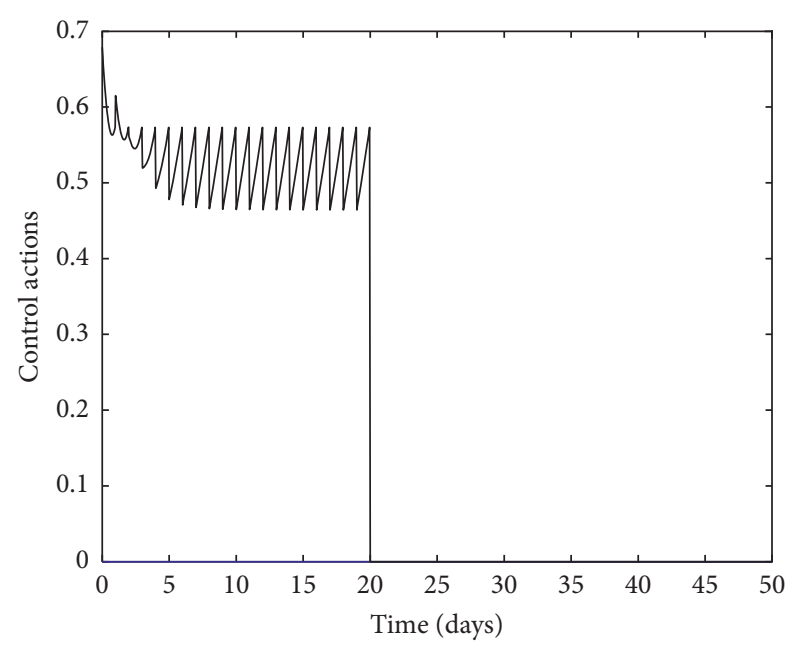

$$
\begin{array}{r}
-V \\
-T
\end{array}
$$

FIGURE 3: Vaccination and treatment efforts constrained to be nonnegative.

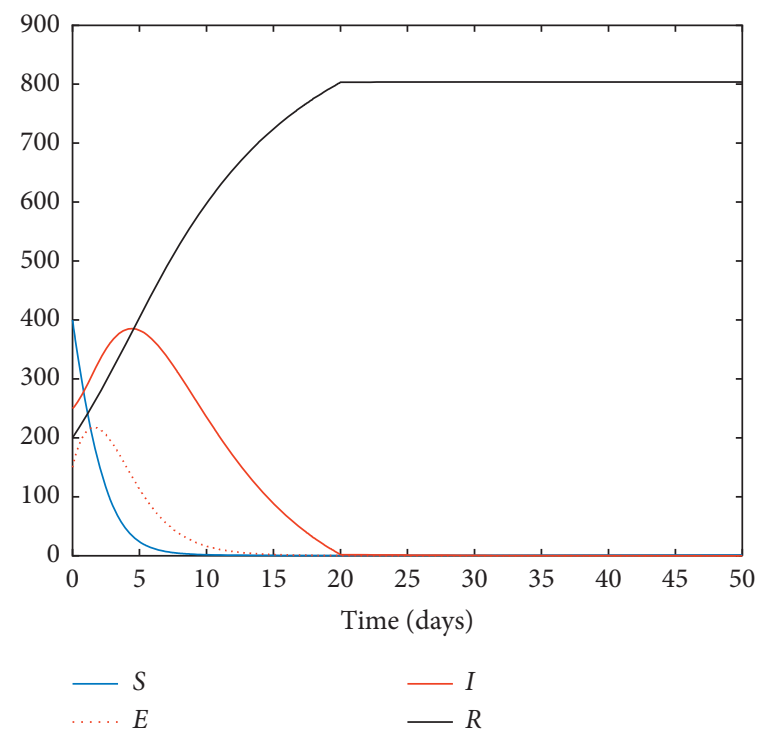

FIgURE 4: Evolution of the populations with the nonnegative control actions.

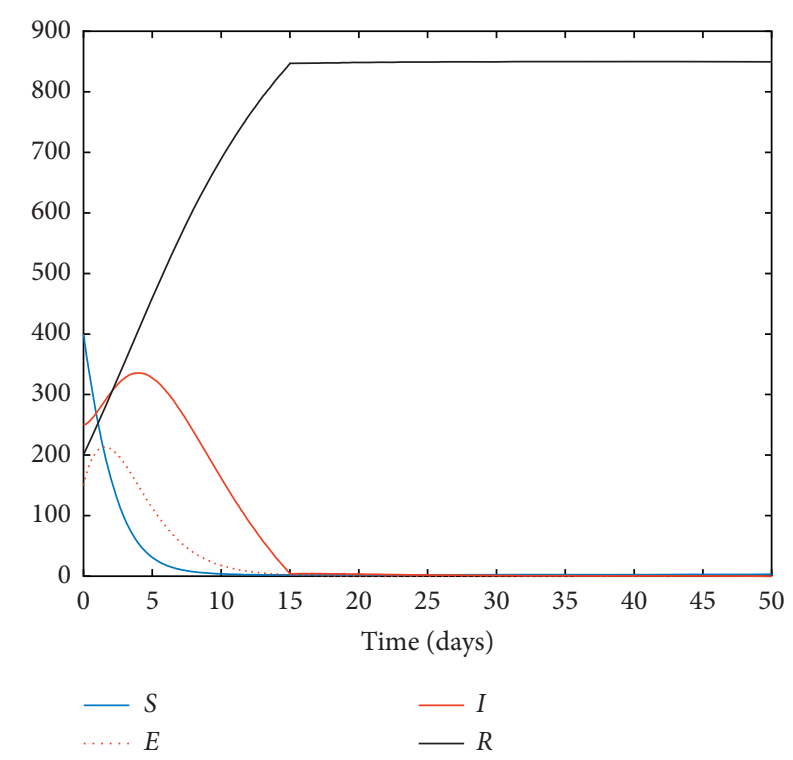

FIgURE 5: Evolution of the populations with the nonnegative control actions and $n=15$ days.

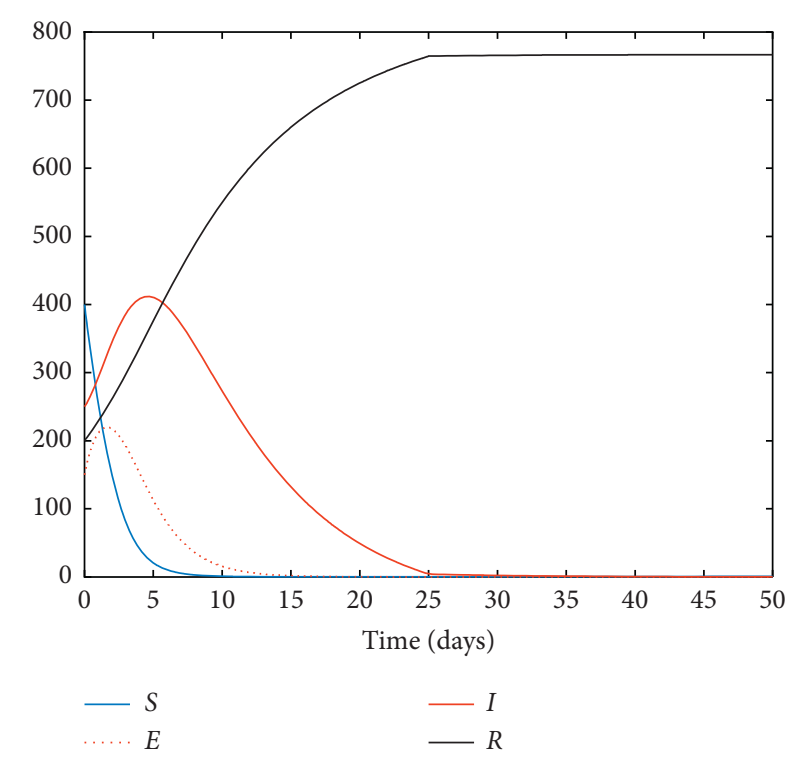

FIGURE 6: Evolution of the populations with the nonnegative control actions and $n=25$ days. 


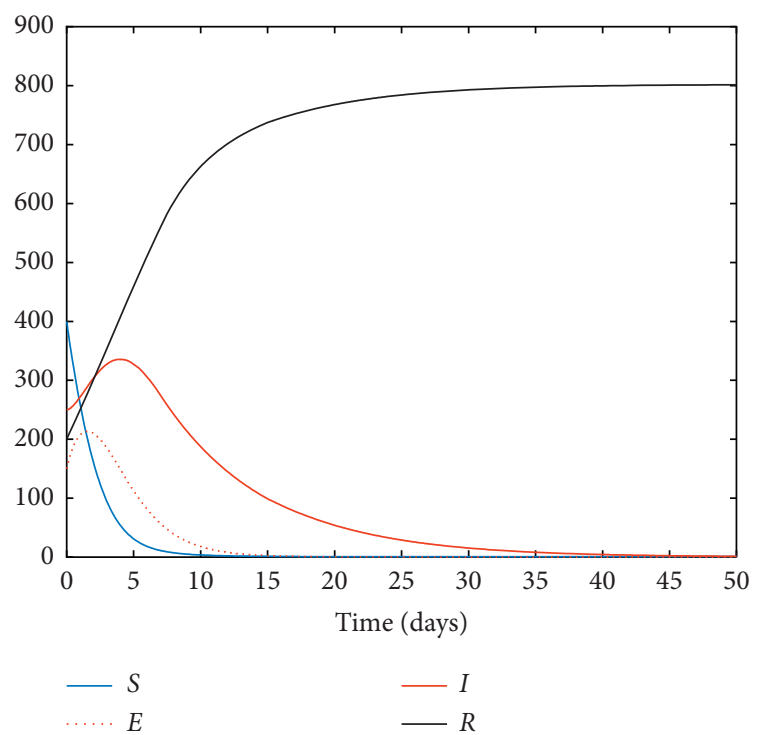

Figure 7: Evolution of the populations with the constrained control actions and $n=15$ days.

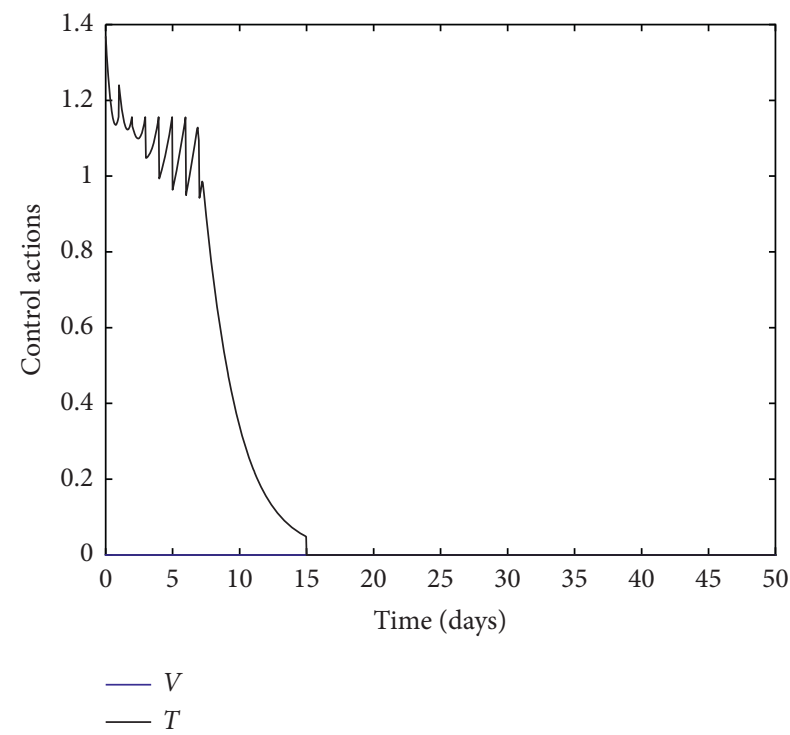

FIGURE 8: Fully constrained vaccination and treatment efforts and $n=15$ days.

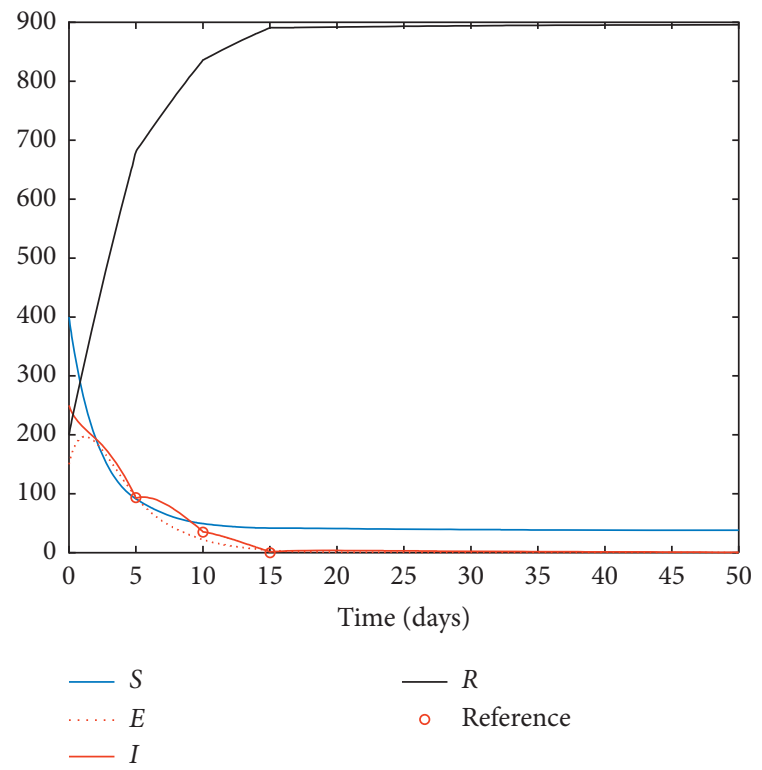

FIgURE 9: Evolution of the closed-loop system for the output targeting procedure.

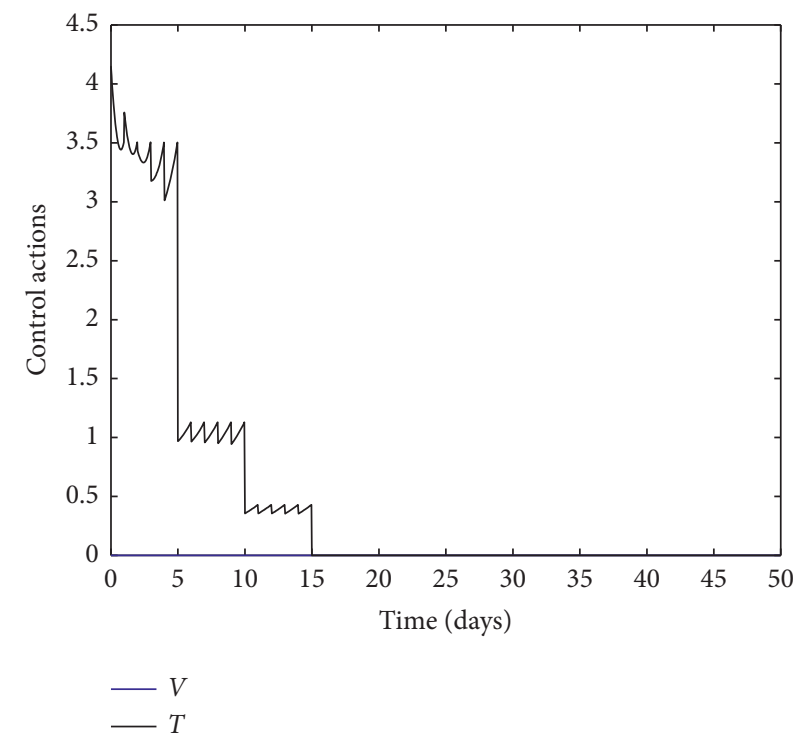

FIGURE 10: Control efforts for the output targeting procedure. 


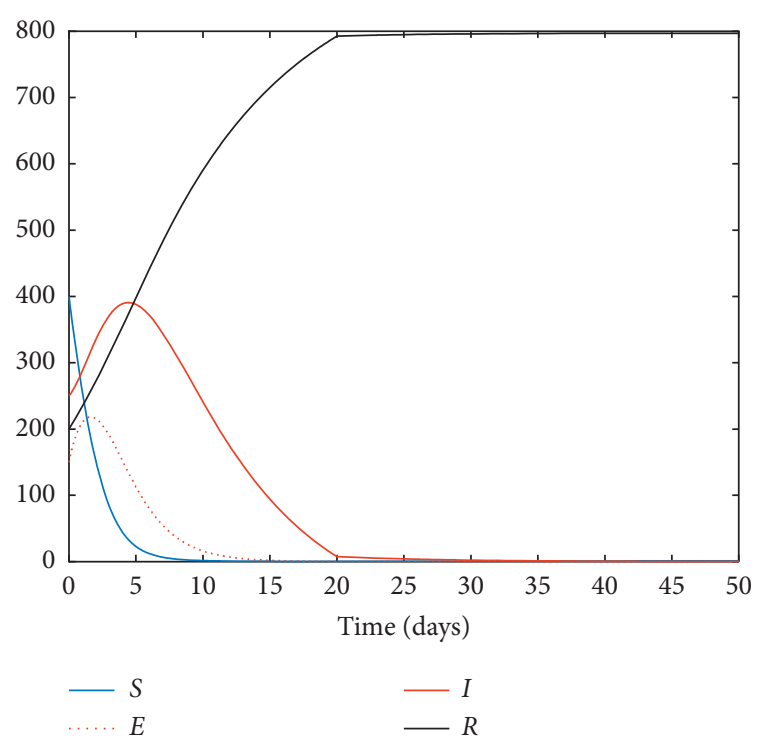

FIGURE 11: Evolution of the system when $\Delta t=12$ hours and $n=20$ days.

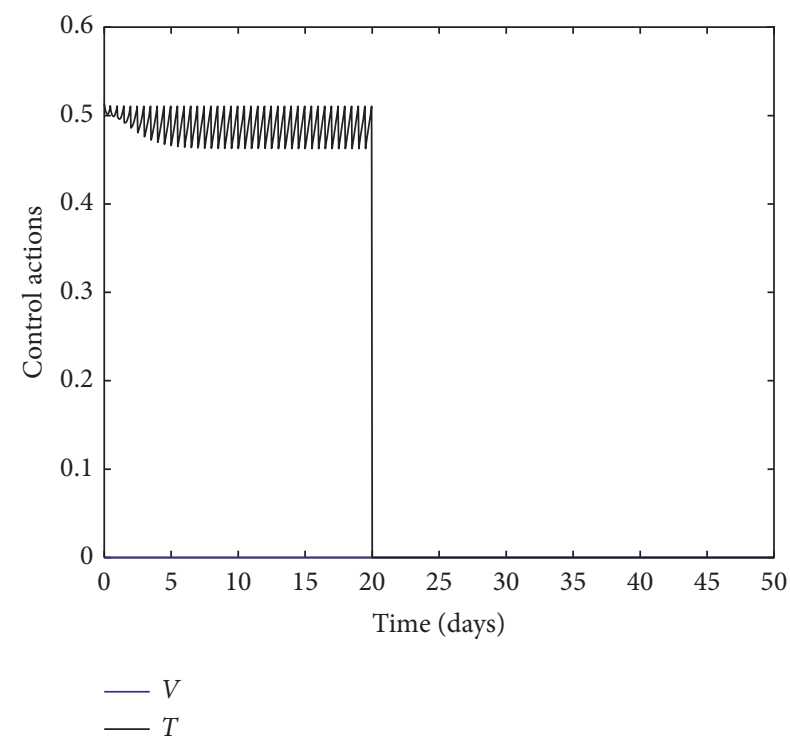

FIGURE 12: Control efforts when $\Delta t=12$ hours and $n=20$ days.

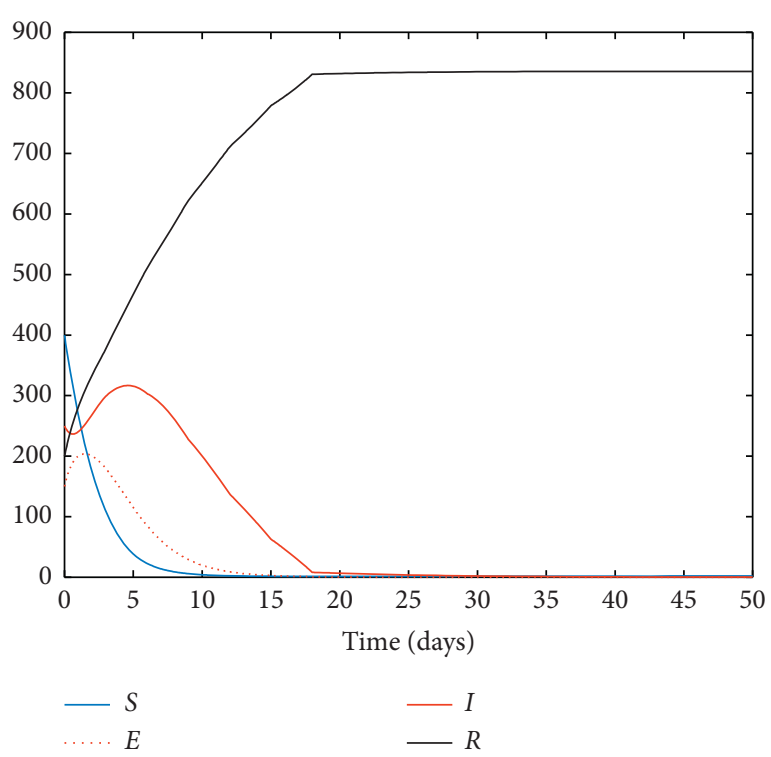

FIgURE 13: Evolution of the system when $\Delta t=72$ hours and $n=20$ days.

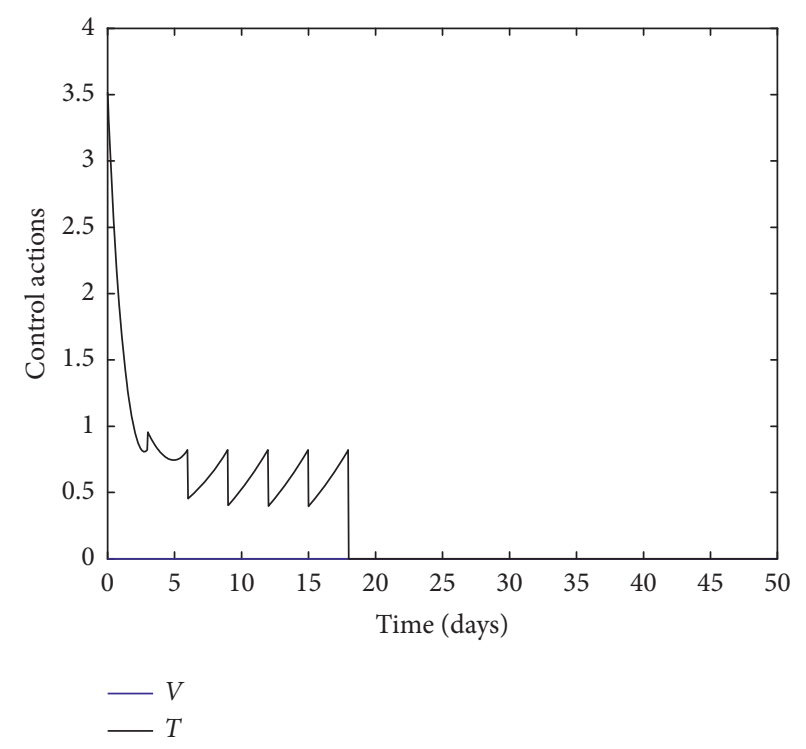

Figure 14: Control efforts when $\Delta t=72$ hours and $n=20$ days. 


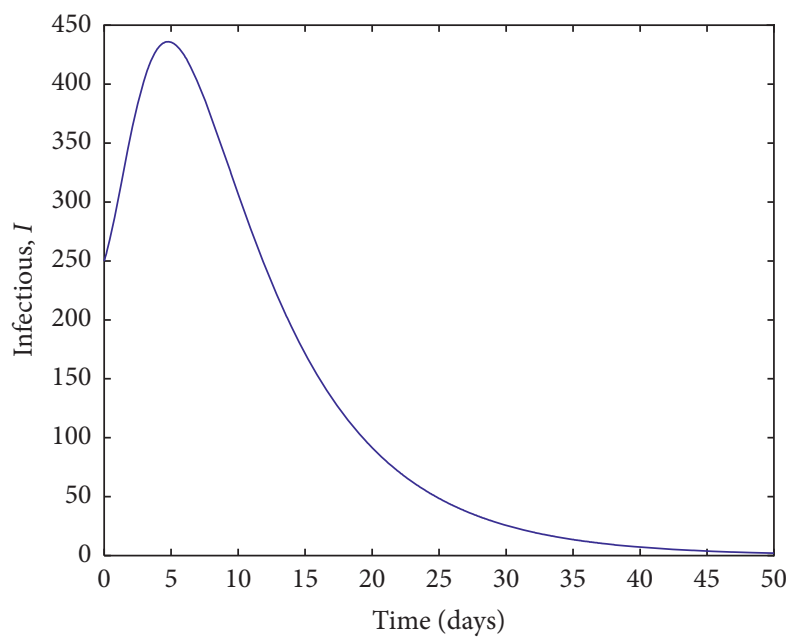

Figure 15: Evolution of the infectious in the absence of external actions.

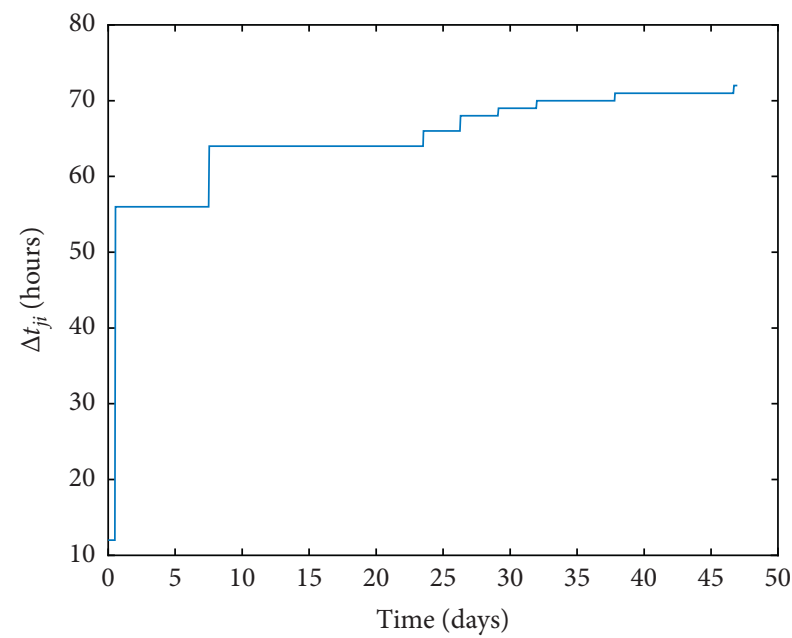

FIgURE 16: Evolution of $\Delta t$.

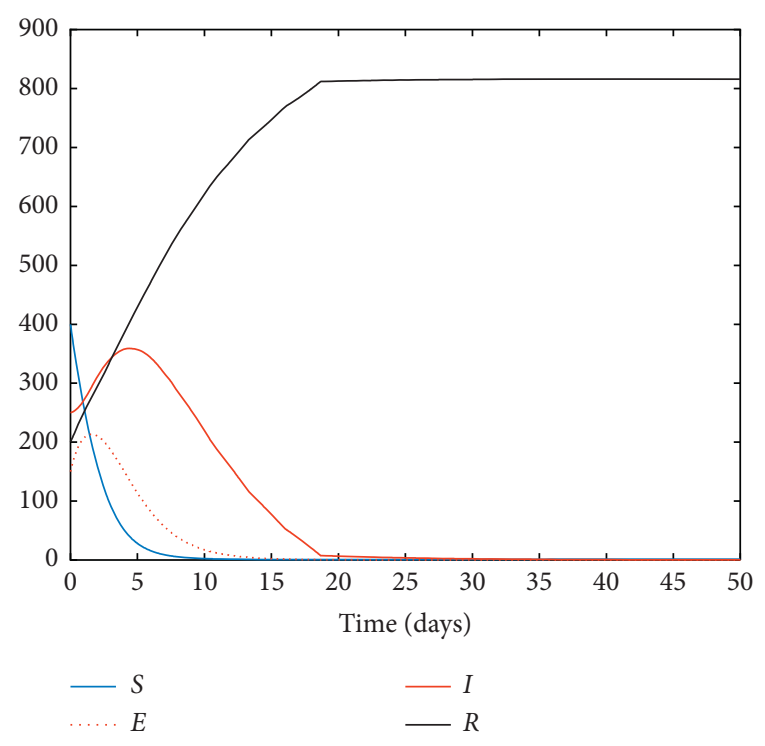

Figure 17: Evolution of the closed-loop system when the adaptive small sampling time procedure is employed.

$$
\left.\begin{array}{c}
T_{a 0}=t_{0}=t, \\
t_{1}=T_{a 0}+\Delta t_{00}, \ldots, t_{n-1}=T_{a 0}+\sum_{i=0}^{n-1} \Delta t_{0 i}, \\
t_{n}=\sum_{j=0}^{1} T_{a j}=t+\sum_{i=0}^{n} \Delta t_{0 i}, \\
t_{n+1}=\sum_{j=0}^{1} T_{a j}+\Delta t_{10}, \ldots, t_{2 n-1}=\sum_{j=0}^{1} T_{a j}+\sum_{i=0}^{n-1} \Delta t_{1 i} t_{2 n}=\sum_{j=0}^{2} T_{a j}=T_{a 0}+T_{a 1}+\sum_{i=0}^{n} \Delta t_{1 i}=t+\sum_{j=0}^{2} \sum_{i=0}^{n} \Delta t_{j i}, \\
t_{2 n+1}=t_{2 n}+\Delta t_{20} t_{3 n}=\sum_{j=0}^{3} T_{a j}=t+\sum_{j=0}^{3} \sum_{i=0}^{n} \Delta t_{j i} t_{\ell n}=t+\sum_{j=0}^{n} \sum_{i=0}^{n} \Delta t_{j i} .
\end{array}\right\}
$$


4.3.2. Adaptation of the Larger Sampling Period. A faster implementation alternative with lower computational cost is concerned with the adaptation of the larger sampling period. The proposed rule is of a similar structure to that of the above one, (65) and (66), as follows:

$$
\begin{gathered}
\Delta t_{j i}=\frac{T_{a j}}{n}, \quad i=0,1, \ldots, n-1 j=1,2, \ldots, \ell, \\
T_{a, j+1}=T_{a j}+\max \left(T_{m}, \frac{C_{1}}{C_{2}+C_{3}\left\|\dot{y}\left(T_{a j}\right)-\dot{y}\left(T_{a, j-1}\right)\right\|}\right), \\
j=1,2, \ldots, \ell
\end{gathered}
$$

with design constants $C_{i}>0, i=1,2,3$, which satisfy $\left(C_{1} / C_{2}\right)=T_{M}$, where $T_{M} \geq\left(T_{m}\right)$ and $T_{m}>0$ are, respectively, the maximum and minimum prescribed values for the larger sampling period of the sequence $\left\{T_{a, j+1}-T_{a j}\right\}_{j=0}^{\ell}$ got from (68).

\section{Numerical Examples}

This section contains some numerical simulation examples illustrating the theoretical results presented in the previous sections. Thus, consider the SEIR model (1)-(4) with parameters given by $b=(1 / 80), b=180 b=180$ years $^{-1}$, $d=(1 / 70)$ years $^{-1}, \quad c=0.0017, \quad f=(1 / 2.2), \quad a=0.0455$, $c=0.0017, f=(1 / 2.2), g=(1 / 12.2)$, all of which in unit of days $^{-1}$. The initial conditions are $S(0)=400, E(0)=150$, $I(0)=250, R(0)=200$. In the absence of any external control action, the evolution of the system is displayed in Figure 1. It can be observed that all the subpopulations remain nonnegative for all time as claimed by Theorem 1. Moreover, it can be concluded from Figure 1 that the infection vanishes in a natural way without the action of external agents. However, the infection remains in the society for around 40 days. This is a typical situation for many infections, such as SARS$\mathrm{CoV}-2$, which vanishes asymptotically but affects a huge fraction of society during a long period of time. In order to reduce the presence of infection, vaccination and treatment are introduced in the model and designed according to the controllability approach introduced in Section 4.

In this way, we consider as system's output $y(t)=I(t)=$ $\left[\begin{array}{llll}0 & 0 & 1 & 0\end{array}\right] x(t)$ with $x(t)^{T}=\left[\begin{array}{llll}S(t) & E(t) & I(t) & R(t)\end{array}\right]$ and the values of $\Delta t=24$ hours, $n=20$ days, and $y^{*}=0$. In particular, $\Delta t$ is the period which parameterizes (46), generalized in (67), for the adaptive case. These values mean that we would like the eradication of the infectious individuals to be in 20 days instead of in the original 40 days. Thus, Theorem 6 is employed to design the vaccination and treatment efforts through (54). The control actions given by (54) are depicted in Figure 2, where they are considered as zero for times larger than $n=20$ days.

The Gramian is a scalar in this case and takes the value of $\left(-1.4 \cdot 10^{4}\right)$ implying that the system is $(20 \mathrm{~d}, 24 \mathrm{~h})$-step-tostep output-reachable through this control, according to Theorem 6. It can be observed in Figure 2 that the vaccination effort is negative, which corresponds to a nonrealistic situation. Thus, in order to solve the above problem, the so-obtained control commands are redesigned by saturating them to zero when their value is negative. When this positivity constraint is added to the control efforts, Figure 3 is obtained while the evolution of the closedloop system is displayed in Figure 4.

From Figure 4, it can be deduced that the nonnegative control signals are able to drive the infectious individuals from their initial state to zero in the prescribed time of 20 days. In this example, the control objective is achieved adequately by only using the treatment action while the vaccination remains at zero at all time. This happens in this example since the dynamics of the susceptible and exposed is much faster than the dynamics of the infectious, as revealed in Figure 1. Figures 5 and 6 show the evolution of the closedloop system for the cases when $n=15$ and $n=25$, respectively. The controllability approach developed in Section 4 allows calculating the control efforts straightforwardly from the specifications, which is one of the great advantages of the presented frame, providing a design method to directly achieve the desired objective.

It can also be observed in Figures 4-6 that the closedloop system remains nonnegative for all time just imposing the nonnegativity of the control commands. However, from a theoretical point of view, we have to consider constraints (56) and (57), containing upper-bounds, in order to guarantee the positivity of the system. When the upper-bounds for the control signals are imposed, the evolution of the closed-loop system is depicted in Figure 7, while the constrained control commands are given in Figure 8 for the case $n=15$ days.

It can be deduced from Figures 5 and 7 that the addition of the upper-bounding in the control actions worsens the performance of the control system, requiring a larger period to eradicate the infectious individuals. Therefore, the theoretical upper-bounds of the control commands are too conservative and should be avoided when the system is nonnegative in practice. Moreover, Figures 4-6 show a peak in the infectious individuals curve. This peak is not often desired in reality. Thus, the output targeting procedure presented in Section 4.3 is now used in order to change the shape of the infectious curve. To this end, we consider the situation when we want to eradicate the infectious in 15 days, while at 5 and 10 days the desired values for the infectious are given by the sampling of the exponentially decreasing function $I_{\text {ref }}(t)=I_{0} e^{(-t / c)}$ with $c=5$ days. In this way, every $T_{a}=5$ days, the desired values for the infectious are provided. Figure 9 shows the evolution of the closed-loop system while Figure 10 shows the control action.

From Figure 9 it can be deduced that the infectious curve does not exhibit a peak now and tracks well the prescribed reference samples at the expense of a higher treatment effort (Figure 10). Furthermore, the susceptible do not converge to zero now as can be observed in Figure 9 (which is not required in this set-up since we only force the infectious to converge to zero). In this example, the control efforts are maintained nonnegative (by applying saturation to zero for negative values), but the upper-bounding has not been 
considered. Finally, the evolution of the system for different values of $\Delta t$ is analyzed now. Thus, Figures 11 and 12 display the evolution and control efforts, respectively, of the system when $\Delta t=12$ hours, and Figures 13 and 14 depict the evolution and control efforts, respectively, when $\Delta t=72$ hours and $n=20$ days.

It is deduced from Figures 11 and 13 that the closed-loop performance worsens as $\Delta t$ increases, as would be expected, since the infectious are not exactly zero at 20 days when $\Delta t=72$. However, the results obtained are not so different as would have been expected. The reason for this relies on the behavior of the infectious curve for the control-free case, depicted in Figure 15 for convenience. In Figure 15, it can be seen that the infectious exhibit a behavior pretty similar to a straight line in the uprising and decreasing sectors. Therefore, the linear approximation in these sectors provides appropriate values for the control calculations. This reason explains the great performance obtained when applying the calculated control commands in the presented examples. The curve differs from the straight line for small values of the infectious. Thus, the worst behavior for $\Delta t=72$ hours is shown in Figure 13 near to 20 days, which corresponds to small values of the infectious.

Figure 16 displays the adaptation of the small sampling time, $\Delta t$, according to (64) for $K_{1}=72, K_{2}=K_{3}=1$ and $\Delta t_{m}=12 \mathrm{~h}, \Delta t_{M}=72$ hours. It can be seen in Figure 16 that the initial sampling time is 12 hours and the algorithm selects the sampling time according to the proposed algorithm. The closed-loop system obtained when adapting the small sampling time is shown in Figure 17. Since the sampling time converges to large values, the obtained performance is similar to Figure 13, corresponding to the case of $\Delta t=72$ hours.

In conclusion, the controllability-based approach allows designing the control efforts by directly taking into account the desired objective. This is a great advantage of the design method since it allows guaranteeing a prescribed performance for the closed-loop based on a real situation. The control commands are saturated in order to guarantee the nonnegativity of the subpopulations. The nonnegativity of the control actions is required in order to avoid nonphysical situations, but the theoretically calculated upper-bounds are too conservative in practice. It is of interest to extend the above results in the future to the stochastic context by considering perturbations in the equilibrium points and uncertainties in the data. See, for instance, [31, 32].

\section{Conclusions}

This manuscript has studied an SEIR epidemic model which is eventually subject to vaccination and treatment controls. Its main properties concerning the solution boundedness for finite initial conditions, as well as the solution nonnegativity for all time under any finite nonnegative initial conditions and its equilibrium points, have been investigated. Later on, the output-reachability aspects on the linearization along the trajectory of a particular model of the previous general one are investigated. The measurable output to be approximately matched to follow a suitable prescribed sampled trajectory is defined, necessarily fulfilling the constraint of having a smaller dimension than four, which is the dimension of the state of the SEIR epidemic model. This constraint is an "a priori" necessary condition for output-reachability. The output solution is approximated by a point-to-point linearization via an updated sampled Jacobian matrix through a set of samples. Such samples can be optionally selected as driven by a constant sampling period or with a time-varying one which is given by an adaptive sampling rule which takes into account the time-derivative values of the output while being decreased (respectively, increased) as the output varies faster (respectively, slower). Finally, the formal aspects have been illustrated through discussed numerical examples.

\section{Data Availability}

No data were used to support this study.

\section{Conflicts of Interest}

The authors declare that they have no conflicts of interest regarding the publication of this article.

\section{Acknowledgments}

The authors are grateful to the Spanish Government for Grants RTI2018-094336-B-I00 and RTI2018-094902-B-C22 (MCIU/AEI/FEDER, UE) and to the Basque Government for Grant IT1207-19. They also thank the Spanish Institute of Health Carlos III for its support through Grant COV 20/ 01213.

\section{References}

[1] K. Yang, E. Wang, Y. Zhou, and K. Zhou, "Optimal vaccination policy and cost analysis for epidemic control in resource-limited settings," Kybernetes, vol. 44, no. 3, pp. 475-486, 2015.

[2] L. F. Nie and Y. N. Xue, "The roles of maturation delay and vaccination on the spread of Dengue virus and optimal control," Advances in Difference Equations, vol. 2017, p. 278, 2017.

[3] C.P. Bhunu, M. Masocha, and C. W. Mahera, "Effects of preexposure vaccination and quarantine in the fight against Ebola,” Cogent Biology, vol. 2, Article ID 1199176, 2016.

[4] J. Wu, R. Dhingra, M. Gambhir, and J. V. Remais, "Sensitivity analysis of infectious disease models: methods, advances and their application," Journal of the Royal Society Interface, vol. 10, no. 86, Article ID 20121018, 2013.

[5] H. Zhang, Z. Yang, K. A. Pawelek, and S. Liu, "Optimal control strategies for a two-group epidemic model with vaccination-resource constraints," Applied Mathematics and Computation, vol. 371, Article ID 124956, 2020.

[6] K. Lata, S. N. Mishra, A. K. Misra et al., "An optimal control problem for carrier dependent diseases," Biosystems, vol. 187, no. 1, Article ID 104039, 2021.

[7] M. R. de Pinho, I. Kornienko, and H. Maurer, "Optimal control of a SEIR model with mixed constraints cost," in Proceedings of the 11th Portuguese Conference on Automatic Control (Controlo' 2014), pp. 135-145, Porto, Portugal, July 2014. 
[8] M. H. A. Biswas, L. T. Paiva, and M. D. R. de Pinho, "A SEIR model for control of infectious diseases with constraints," Mathematical Biosciences and Engineering, vol. 11, no. 4, pp. 761-784, 2014.

[9] R. Nistal, M. De la Sen, S. Alonso-Quesada, and A. Ibeas, "On an SEIADR epidemic model with vaccination, treatment and dead-infectious corpses removal controls," International Journal of Innovative Computing, Information and Control, vol. 15, no. 6, pp. 2053-2067, 2019.

[10] M. De la Sen, S. Alonso-Quesada, A. Ibeas, and R. Nistal, "On a SEIADR epidemic model with vaccination, treatment and dead-infectious corpses removal controls," Mathematics and Computers in Simulation, vol. 163, pp. 47-79, 2019.

[11] I. H.I. Ahmed, P. J. Witbooi, and K. Patidar, "Modeling the dynamics of an epidemic under vaccination in two interacting populations," Journal of Applied Mathematics, vol. 2012, p. 14, Article ID 275902, 2012.

[12] K. Li, M. Small, H. Zhang, and X. Fu, "Epidemic outbreaks on networks with effective contacts," Nonlinear Analysis. Real World Applications, vol. 11, no. 2, pp. 1017-1025, 2010.

[13] M. De la Sen, A. Ibeas, S. Alonso-Quesada, and R. Nistal, “On a SIR model in a patchy environment under constant and feedback decentralized controls with asymmetric parameterizations," Symmetry, vol. 11, no. 3, Article ID 430, 2019.

[14] M. De la Sen, "Parametrical non-complex tests to evaluate partial decentralized linear-output feedback control stabilization conditions for their centralized stabilization counterparts," Applied Sciences-Basel, vol. 9, no. 9, Article ID 1739, 2019.

[15] L. A. Meyers, "Contact network epidemiology: bond percolation applied to infectious disease prediction and control," Journal of Mathematical Biology, vol. 44, no. 1-2, pp. 63-86, 2007.

[16] A. Iggidr and M. O. Souza, "State estimators for some epidemiological systems," Mathematical Biology, vol. 78, pp. 225-256, 2019.

[17] Q. Cui, Z. Qiu, W. Liu, and Z. Hu, "Complex dynamics of an SIR epidemic model with nonlinear saturated incidence and recovery rate," Entropy, vol. 2017, no. 19, pp. 1-16, Article ID 305, 2017.

[18] E. Y. Erten, I.-N. Lizier, M. Piraveenan, and M. Prokopenko, "Criticality and information dynamics in epidemiological models," Entropy, vol. 2017, no. 19, pp. 1-11, Article ID 194, 2017.

[19] T. Caraco and N. Wang, "Free-living pathogens: life-history constraints and strain competition," Journal of Theoretical Biology, vol. 250, no. 3, pp. 569-579, 2008.

[20] M. De la Sen, "On the approximated reachability of a class of time-varying systems based on their linearized behaviour about the equilibria: applications to epidemic models," Entropy, vol. 2019, no. 21, pp. 1-31, Article ID 1045, 2019.

[21] M. De la Sen, "On the design of hyperstable feedback controllers for a class of parameterized nonlinearities. Two application examples for controlling epidemic models," International Journal of Environmental Research and Public Health, vol. 16, no. 15, Article ID 2689, 2019.

[22] T. De la Sen and S. Alonso-Quesada, "Control issues for the Beverton-Holt equation in ecology by locally monitoring the environment carrying capacity: non-adaptive and adaptive cases," Applied Mathematics and Computation, vol. 215, no. 7, pp. 2616-2831, 2009.

[23] T. C. Hsia, "Comparisons of adaptive sampling control laws," IEEE Transactions on Automatic Control, vol. 17, no. 6, pp. 830-821, 1972.
[24] M. Delasen, "Application of the non-periodic sampling to the identifiability and model matching problems in dynamic systems," IEEE Transactions on Automatic Control, vol. 19, no. 1, pp. 29-42, 1974.

[25] M. Delasen, "Application of the non-periodic sampling to the identifiability and model-matching problems in dynamic systems," International Journal of Systems Science, vol. 14, no. 4, pp. 367-383, 1983.

[26] M. Delasen, "A method for improving the adaptation transient using adaptive sampling," International Journal of Control, vol. 40, no. 4, pp. 639-665, 1984.

[27] M. Delasen, "Adaptive sampling for improving the adaptation transients in hybrid adaptive control," International Journal of Control, vol. 41, no. 5, pp. 1189-1205, 1985.

[28] M. Miskowicz, "Send-on-delta concept: an event-based data reporting strategy," Sensors, vol. 6, no. 1, pp. 49-63, 2006.

[29] M. Miskowicz, "Asymptotic effectiveness of the event-based sampling according to the integral criterion," Sensors, vol. 7, no. 1, pp. 16-37, 2007.

[30] M. Miskowicz, "Efficiency of event-based sampling according to error energy criterion," Sensors, vol. 10, no. 3, pp. 2242-2261, 2010.

[31] L. Shaikhet, "Improving stability conditions for equilibria of SIR epidemic model with delay under stochastic perturbations"” Mathematics, vol. 8, no. 8, p. 1302, 2020.

[32] L. Shaikhet and H. Inaba, "Possible effects of mixed prevention strategy for COVID-19 epidemic: massive testing, quarantine and social distancing," in Lyapunov Functionals and Stability of Stochastic Functional Differential EquationsSpringer, Heidelberg, Germany, 2013.

[33] T. Kuniya and H. Inaba, "Possible effects of mixed prevention strategy for COVID-19 epidemic: massive testing, quarantine and social distance," AIMS Public Health, vol. 7, no. 3, pp. $490-503,2020$. 